\title{
Differential gene expression profiling of the sciatic nerve in type 1 and type 2 diabetic mice
}

\author{
YU GU* , ZHUO-LIN QIU* , DE-ZHAO LIU, GUO-LIANG SUN, YING-CHAO GUAN, ZI-QING HEI and XIANG LI \\ Department of Anesthesiology, The Third Affiliated Hospital of Sun Yat-sen University, \\ Guangzhou, Guangdong 510630, P.R. China
}

Received February 26, 2018; Accepted July 17, 2018

DOI: $10.3892 /$ br.2018.1135

\begin{abstract}
Diabetic peripheral neuropathy (DPN) is a common complication of diabetes mellitus (DM). The pathogenic mechanisms of DPN and the therapeutic interventions required may be distinct between type 1 (T1) and type 2 (T2) DM. However, the molecular mechanisms underlying the pathogenesis of DPN in both types of diabetes remain unclear. The aim of the current study was to identify the changes in genes and pathways associated with DPN in sciatic nerves of T1- and T2DM mice using bioinformatics analysis. The microarray profiles of sciatic nerves of T1DM (GSE11343) and T2DM (GSE27382) mouse models were downloaded from the Gene Expression Omnibus database to identify differentially expressed genes (DEGs) in each. DEGs in the two types of DM (with fold change $\geq 2$ and $\mathrm{P}<0.05$ ) were identified with BRB-ArrayTools. Gene Ontology (GO) term and Kyoto Encyclopedia of Genes and Genomes (KEGG) pathway enrichment analyses were performed using the Database for Annotation, Visualization and Integrated Discovery. A protein-protein interaction (PPI) network was constructed using the Search Tool for the Retrieval of Interacting Genes/Proteins and visualized using Cytoscape. Compared with control samples, 623 and 1,890 DEGs were identified in sciatic nerves of T1- and T2DM mice, respectively. Of these, 75 genes were coordinately dysregulated in the sciatic nerves of both models. Many DEGs unique to T1DM mice were localized to the nucleoplasm and were associated with regulation of transcription processes, while many unique to T2DM mice were localized at cell junctions and were associated with ion transport. In addition, certain DEGs may be associated with the different treatment strategies used for the two types of DM. This analysis provides insight into the functional gene sets and
\end{abstract}

Correspondence to: Dr Xiang Li, Department of Anesthesiology, The Third Affiliated Hospital of Sun Yat-sen University, 600 Tianhe Road, Guangzhou, Guangdong 510630, P.R. China

E-mail: lixiang86-26@163.com

*Contributed equally

Key words: type 1 and 2 diabetes mellitus, diabetic peripheral neuropathy, bioinformatics analysis, microarray pathways operating in sciatic nerves in T1- and T2DM. The results should improve understanding of the molecular mechanisms underlying the pathophysiology of DPN, and provide information for the development of therapeutic strategies for DPN specific to each type of DM.

\section{Introduction}

Diabetic peripheral neuropathy (DPN) is a common complication of diabetes mellitus (DM). More than half of patients with DM worldwide suffer from DPN during the course of the disease $(1,2)$. DPN is characterized by distal-to-proximal nerve damage leading to neuropathic pain and loss of sensation (3). It is also associated with notably high morbidity and mortality: Previous studies reported that the mortality rate approximated to $25-50 \%$ within 5-10 years following onset of diabetic neuropathy $(4,5)$. Strict maintenance of normal glycemic level is the only effective treatment available for DPN at present (6).

Though the occurrence of DPN is common to both type 1 (T1) and type 2 (T2)DM, a number of studies have suggested that its pathogenic mechanism may differ between the two $(7,8)$. For example, more abnormalities at the molecular, functional and morphometric levels including increased frequencies of denervated Schwann cells and significant fiber loss have been observed in the peripheral nerve of T1DM mice compared with in T2DM mice $(8,9)$. In addition, different structural changes may lead to variation in nerve conduction velocity for DPN in the two diabetic models (10). These fundamentally different mechanisms for DPN may lead to varying effects of the common treatments, such as in control of glucose, which has been suggested to be more beneficial for DPN in T1DM than in T2DM (7). Therefore, determining the distinct molecular mechanisms underlying DPN in T1- and $\mathrm{T} 2 \mathrm{DM}$ is of paramount importance for the development of successful therapeutic interventions.

In the last decade, the development of high-throughput platforms including microarray technology has allowed researchers to concurrently determine the expression levels of several thousands of differentially expressed genes (DEGs) in diseases $(11,12)$. In addition, the comparison of gene expression profiles of DEGs through microarray technology using bioinformatics analysis has determined distinct pathophysiological mechanisms in different diseases or syndromes including neuropathic pain and chronic radicular 
pain (13). For instance, Zhang et al (14) screened a number of DEGs in a control (healthy) group of mice and a group with streptozotocin (STZ)-induced diabetes, and identified genes co-regulated by both STZ and rosiglitazone, which may be potential targets in the treatment of DPN. However, there are few studies that have compared the gene expression profiles of DEGs between DPN in T1- and T2DM through microarray profiling. In the present study, the aim was to compare the DEGs between the sciatic nerves of T1- and T2DM mouse models by microarray profiling. Furthermore, the distinct biological processes and pathways associated with DPN in T1- or T2DM were analyzed and compared based on gene ontology (GO) and pathway enrichment analyses. This was hoped to provide novel insights into the distinct pathophysiological mechanisms and implicate drug therapies for DPN specific to the different types of DM.

\section{Materials and methods}

Source of microarray data. The gene expression profiles of GSE11343 and GSE27382 were obtained from the Gene Expression Omnibus (GEO) database (http://www.ncbi.nlm. nih.gov/geo/). The annotation platforms for GSE11343 and GSE27382 were GPL1261 and GPL9746, respectively.

For GSE11343 submitted by Wiggin et al (15), included datasets were of five sciatic nerve samples from mice with T1DM induced by STZ (GSM286169, GSM286173, GSM286176, GSM286178 and GSM286430) and four sciatic nerve samples from normal mice (GSM286159, GSM286160, GSM286163 and GSM286165).

For GSE27382 submitted by Pande et al (16), included datasets were of six sciatic nerve samples from $\mathrm{db} / \mathrm{db}$ mice with T2DM (GSM677112-GSM677117) and seven sciatic nerve samples from db/+ (normal) mice (GSM677105-GSM677111).

Pre-processing of microarray data and identification of DEGs. Pre-processing for the cell intensity (CEL) files including conversion into expression measures, background correction and quartile data normalization was performed with BRB-ArrayTools (version 4.5.1) (17). The univariate t-test with a fold change $\geq 2$ and nominal significance level of 0.05 was applied in BRB-ArrayTools to identify the DEGs between the diabetic and normal groups.

Gene ontology and pathway enrichment analyses. To identify the DEGs determined with BRB-ArrayTools, GO and Kyoto Encyclopedia of Genes and Genomes (KEGG) pathway enrichment analyses were performed via the Database for Annotation, Visualization and Integrated Discovery (DAVID 6.8; http://david.abcc.ncifcrf.gov/) $(18,19)$. GO terms [categorized into molecular function (MF), biological process (BP) and cellular component $(\mathrm{CC})$ ] and KEGG pathways with $\mathrm{P}<0.05$ were considered significantly enriched by the DEGs.

Construction of protein-protein interaction (PPI) network and module analysis. With the purpose of evaluating the relationships among DEGs from the perspective of protein interaction, a protein-protein interaction (PPI) network was constructed with the Search Tool for the Retrieval of Interacting Genes/Proteins (STRING 10.5; http://www.string-db.org) and visualized using
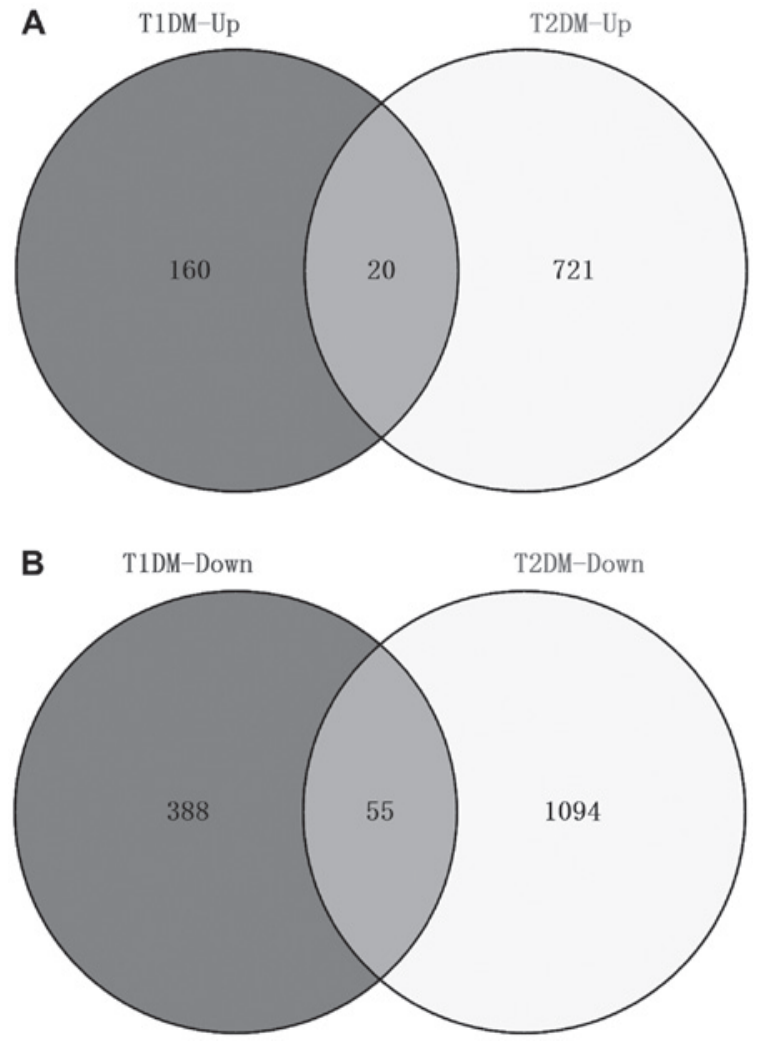

Figure 1. Numbers of (A) upregulated and (B) downregulated differentially expressed genes in the sciatic nerve of T1- and T2DM mouse models. T1DM, type 1 diabetes mellitus; T2DM, type 2 diabetes mellitus.

Cytoscape software (version 3.4.0; http://cytoscape.org/) (18). A combined score $>0.4$ was selected to determine significant interactions among DEGs. In accordance with a previous analysis (20), the connectivity degree of a protein, namely the number of proteins it connected with, was considered to indicate importance of the protein in the PPI network.

Module analysis was performed in the plugin ClusterONE (version 1.0) in Cytoscape with a threshold of $\mathrm{P}<0.001$. In addition, function and pathway enrichment analyses were performed for DEGs in the modules with a threshold of $\mathrm{P}<0.05$.

\section{Results}

Identification of DEGs. Based on the criteria of a nominal significance level of 0.05 and fold change $\geq 2,623$ and 1,890 DEGs were identified in sciatic nerves of T1DM (GSE11343) and T2DM (GSE27382) mice, respectively, as compared with the control samples. Among them, 75 genes were identified to be coordinately dysregulated in sciatic nerves of both models, with 20 genes upregulated and 55 genes downregulated (Fig. 1 and Table SI). Meanwhile, 160 upregulated and 388 downregulated DEGs were unique to T1DM (Fig. 1 and Table SII), and 721 upregulated and 1,094 downregulated DEGs were unique to T2DM (Fig. 1 and Table SIII).

GO and pathway enrichment analyses. There were 60 coincident enriched GO terms for DEGs in T1- and T2DM, the top 20 of which are listed in Table I. In addition, the top 20 enriched GO terms for DEGs that were unique to sciatic nerves from the T1- or T2DM model are presented in Table II. Besides these, 
Table SI. List of genes coordinately regulated in type 1 and type 2 diabetes mellitus.

\begin{tabular}{lccccc}
\hline $\begin{array}{l}\text { Gene } \\
\text { count }\end{array}$ & $\begin{array}{c}\text { Gene } \\
\text { symbol }\end{array}$ & $\begin{array}{c}\text { Gene } \\
\text { count }\end{array}$ & $\begin{array}{c}\text { Gene } \\
\text { symbol }\end{array}$ & $\begin{array}{c}\text { Gene } \\
\text { count }\end{array}$ & $\begin{array}{c}\text { Gene } \\
\text { symbol }\end{array}$ \\
\hline
\end{tabular}

\begin{tabular}{|c|c|c|c|c|c|}
\hline \multicolumn{6}{|c|}{ Upregulated } \\
\hline 1 & C1qtnf9 & 8 & Myo5b & 15 & Hipk3 \\
\hline 2 & Hspb7 & 9 & Tcf 15 & 16 & Hif3a \\
\hline 3 & Fmo2 & 10 & Ltbp2 & 17 & Pde2a \\
\hline 4 & Cyp1a1 & 11 & Tlr7 & 18 & Fkbp5 \\
\hline 5 & Gpihbp1 & 12 & Map3k6 & 19 & 8430436 N08Rik \\
\hline 6 & Mbd1 & 13 & Angptl4 & 20 & Dnah11 \\
\hline 7 & Gm4876 & 14 & D8Ertd82e & & \\
\hline \multicolumn{6}{|c|}{ Downregulated } \\
\hline 1 & Kcna2 & 20 & Pcyox 11 & 39 & Tmem229a \\
\hline 2 & Kif5b & 21 & Rapgef4 & 40 & Rian \\
\hline 3 & $\operatorname{Cct} 4$ & 22 & Sptssb & 41 & $\mathrm{C} 1 \mathrm{ql} 3$ \\
\hline 4 & Fchsd2 & 23 & 5930427L02Rik & 42 & Chic1 \\
\hline 5 & Fgf14 & 24 & Efcab14 & 43 & Cntn3 \\
\hline 6 & Fndc5 & 25 & Frzb & 44 & Map1b \\
\hline 7 & Kcna1 & 26 & Cemip & 45 & Slc35f1 \\
\hline 8 & Slc25a27 & 27 & $\mathrm{Zic} 2$ & 46 & Sox9 \\
\hline 9 & Galnt3 & 28 & Zic1 & 47 & Gpm6b \\
\hline 10 & Ncald & 29 & Slc1a3 & 48 & Slc47a1 \\
\hline 11 & Senp8 & 30 & Snhg14 & 49 & Elavl2 \\
\hline 12 & Asph & 31 & Bmp5 & 50 & Ptn \\
\hline 13 & Pdia3 & 32 & Lamc3 & 51 & Dcc \\
\hline 14 & Bicd1 & 33 & Nipal1 & 52 & Atp13a5 \\
\hline 15 & Elovl4 & 34 & C78859 & 53 & Hpca \\
\hline 16 & Npas3 & 35 & Glipr2 & 54 & Sox 8 \\
\hline 17 & Opcml & 36 & Enah & 55 & Zic5 \\
\hline 18 & $\mathrm{Nr} 2 \mathrm{f} 2$ & 37 & Gm19935 & & \\
\hline 19 & Ntrk3 & 38 & Paqr8 & & \\
\hline
\end{tabular}

21 and 27 KEGG pathways were enriched for DEGs in T1- and T2DM, respectively. Among them, 4 pathways were coincident in both models (Table III), while 17 and 23 pathways were unique to T1- and T2DM respectively (Table IV).

PPInetwork. A total of 419 nodes and 1,343 edges were involved in the PPI for T1DM, whereas 1,416 nodes and 11,077 edges were involved in that for T2DM (data not shown). The top 10 proteins in each of T1- and T2DM with a relatively high degree of connectivity in the PPI network are presented in Table V.

Extent of enriched function and topological structure analysis for the modules of the PPI networks. Based on the module analysis by ClusterONE, a single module was determined in the PPI for T1DM (Fig. 2A) and 7 modules for T2DM (Fig. 2B-H). The information on the modules, including node number, P-value, protein with highest degree of connectivity, and numbers of GO terms and KEGG pathways are shown in Table VI.

\section{Discussion}

DPN is among the most frequent complications for T1- and T2DM. It is generally considered that DPN results from damage by hyperglycemia, regardless of the type of DM (3). In
Table SII. List of genes specific to type 1 diabetes mellitus.

\begin{tabular}{lccccc}
\hline $\begin{array}{l}\text { Gene } \\
\text { count }\end{array}$ & $\begin{array}{c}\text { Gene } \\
\text { symbol }\end{array}$ & $\begin{array}{c}\text { Gene } \\
\text { count }\end{array}$ & $\begin{array}{c}\text { Gene } \\
\text { symbol }\end{array}$ & $\begin{array}{c}\text { Gene } \\
\text { count }\end{array}$ & $\begin{array}{c}\text { Gene } \\
\text { symbol }\end{array}$ \\
\hline
\end{tabular}

Upregulated

\begin{tabular}{|c|c|c|c|c|c|}
\hline 1 & Myh2 & 55 & Wdr63 & 108 & Bbd9 \\
\hline 2 & Doc2b & 56 & Gm17455 & 109 & Klk1b24 \\
\hline 3 & Atxn711 & 57 & Per2 & 110 & Acer2 \\
\hline 4 & Tbata & 58 & Gimap8 & 111 & Angpt2 \\
\hline 5 & Rph3al & 59 & Cpa3 & 112 & ВC003965 \\
\hline 6 & Apoc3 & 60 & C77370 & 113 & Rtp3 \\
\hline 7 & $\mathrm{Nr} 5 \mathrm{a} 2$ & 61 & Rgs3 & 114 & Iffo1 \\
\hline 8 & Alpk3 & 62 & Oosp1 & 115 & Lhx6 \\
\hline 9 & Tal1 & 63 & Dbp & 116 & Tmem88 \\
\hline 10 & Aldh3b2 & 64 & Dl14 & 117 & 4930539E08Rik \\
\hline 11 & Rasd2 & 65 & Mapk12 & 118 & Adcyap1r1 \\
\hline 12 & Iigp1 & 66 & D7Bwg0826e & 119 & Anapc11 \\
\hline 13 & Etnppl & 67 & Myct1 & 120 & Clca3a2 \\
\hline 14 & Colec11 & 68 & Ranbp17 & 121 & Cyyr1 \\
\hline 15 & Ifi44 & 69 & Adcy4 & 122 & Lrpap1 \\
\hline 16 & Krt18 & 70 & Shisa6 & 123 & Cdh13 \\
\hline 17 & Gimap4 & 71 & Asxl1 & 124 & Abcg 1 \\
\hline 18 & Adgrf5 & 72 & Ppp1r3d & 125 & Syt7 \\
\hline 19 & Spns2 & 73 & Camk2n1 & 126 & Jag2 \\
\hline 20 & Cabp4 & 74 & Mboat1 & 127 & Odf $3 b$ \\
\hline 21 & D7Wsu130e & 75 & Arrdc4 & 128 & Rilp \\
\hline 22 & Ms4a2 & 76 & Ctsg & 129 & Sox 7 \\
\hline 23 & Exос 312 & 77 & Pvr & 130 & 9430030 N17Rik \\
\hline 24 & Ms4a8a & 78 & Tox & 131 & Adgrl4 \\
\hline 25 & Csf2rb2 & 79 & 4833438C02Rik & 132 & Usp3 \\
\hline 26 & Ace2 & 80 & Rasgrf2 & 133 & Vps51 \\
\hline 27 & Itih4 & 81 & Tnfsf13os & 134 & Kcnq1 \\
\hline 28 & Robo4 & 82 & Gm11772 & 135 & Krt20 \\
\hline 29 & Gm11716 & 83 & 4833411I10Rik & 136 & Rabgap11 \\
\hline 30 & 4921507P07Rik & 84 & Mfng & 137 & Sphk2 \\
\hline 31 & Gp1bb & 85 & Sox17 & 138 & 1810021B22Rik \\
\hline 32 & Vwf & 86 & D930003E18Rik & 139 & 4930447M23Rik \\
\hline 33 & Erg & 87 & Rtn4r12 & 140 & Arhgef15 \\
\hline 34 & Eng & 88 & Kank3 & 141 & Cpsf1 \\
\hline 35 & Mett122 & 89 & Ptprb & 142 & Foxp4 \\
\hline 36 & Lama3 & 90 & Rpusd2 & 143 & Sema5b \\
\hline 37 & 5730458M16Rik & 91 & Sigirr & 144 & Actr1b \\
\hline 38 & 4833403J16Rik & 92 & Trim47 & 145 & Hsd17b11 \\
\hline 39 & Tceanc2 & 93 & Adamdec 1 & 146 & Myrip \\
\hline 40 & Fam107a & 94 & Cgrrf1 & 147 & Gimap5 \\
\hline 41 & Slc $27 \mathrm{a} 2$ & 95 & Tigd3 & 148 & Prkcg \\
\hline 42 & Zfpm1 & 96 & Spn & 149 & Abi3 \\
\hline 43 & Clec14a & 97 & Car7 & 150 & Bmp4 \\
\hline 44 & Oasl1 & 98 & Mettl13 & 151 & Mgst2 \\
\hline 45 & Anxa8 & 99 & Rap1gap & 152 & Ppp1r131 \\
\hline 46 & D130037M23Rik & 100 & 2210019G11Rik & 153 & Selo \\
\hline 47 & Fam189a1 & 101 & 6330403A02Rik & 154 & Sema3f \\
\hline 48 & Clca3a1 & 102 & Tmprss2 & 155 & Mocs1 \\
\hline 49 & Mmrn2 & 103 & $\mathrm{Ak} 8$ & 156 & Nos3 \\
\hline 50 & Cbfa2t3 & 104 & Cyp11a1 & 157 & Pold1 \\
\hline 51 & Notch4 & 105 & Ushbp1 & 158 & AU022252 \\
\hline 52 & She & 106 & Slc6a7 & 159 & D630045J12Rik \\
\hline 53 & Soat2 & 107 & 6330416G13Rik & 160 & Ngef \\
\hline 54 & Unc45bos & & & & \\
\hline
\end{tabular}

Downregulated

$\begin{array}{llrlll}1 & \text { Ccnt2 } & 131 & \text { 9530029O12Rik } & 260 & \text { Dag1 } \\ 2 & \text { Col4a5 } & 132 & \text { A330075M08Rik } 261 & \text { Itm2a } \\ 3 & \text { Crk1 } & 133 & \text { Abce1 } & 262 & \text { Luc712 } \\ 4 & \text { Dnajc18 } & 134 & \text { Anp32a } & 263 & \text { Marcks } \\ 5 & \text { Ebf2 } & 135 & \text { Arhgef6 } & 264 & \text { Nktr } \\ 6 & \text { Lamb1 } & 136 & \text { Chl1 } & 265 & \text { Saa1 } \\ 7 & \text { Pbrm1 } & 137 & \text { Cp } & 266 & \text { Senp6 } \\ 8 & \text { R3hdm4 } & 138 & \text { Ctse } & 267 & \text { Serinc1 } \\ 9 & \text { Rab10 } & 139 & \text { Dcbld2 } & 268 & \text { Sfrp1 }\end{array}$


Table SII. Continued.

\begin{tabular}{lccccc}
\hline $\begin{array}{l}\text { Gene } \\
\text { count }\end{array}$ & $\begin{array}{c}\text { Gene } \\
\text { symbol }\end{array}$ & $\begin{array}{c}\text { Gene } \\
\text { count }\end{array}$ & $\begin{array}{c}\text { Gene } \\
\text { symbol }\end{array}$ & $\begin{array}{c}\text { Gene } \\
\text { count }\end{array}$ & $\begin{array}{c}\text { Gene } \\
\text { symbol }\end{array}$ \\
\hline
\end{tabular}

\begin{tabular}{llllll}
\hline 10 & Rbfox2 & 140 & Eif4a1 & 269 & Slc25a24 \\
11 & Rs124d1 & 141 & Epb4112 & 270 & Snx18 \\
12 & Sat1 & 142 & Far1 & 271 & Snx5 \\
13 & Slc25a3 & 143 & Gatm & 272 & Tbl1xr1 \\
14 & Smarcc1 & 144 & Gpbp111 & 273 & Zic4 \\
15 & Spry2 & 145 & Hba-a2 & 274 & 9930031P18Rik \\
16 & Suz12 & 146 & Hmgcr & 275 & Akap13
\end{tabular}

17 Timp2

$18 \quad$ Zfp626

19 Actb

20 Amd1

21 Arf1

22 Arhgap29

23 Atp6v1b2

24 C030046G05

25 Cbx 5

$26 \quad \mathrm{Cd} 47$

27 Cryzl1

28 D2Ertd640e

29 Elavl1

30 Hist1h1c

31 Il6st

32 Katnbl1

33 Lats2

34 P4ha1

35 Pi4kb

36 Plekhg 1

37 Polb

38 Pten

39 Rbbp4

$40 \quad$ Rnf13

$41 \quad$ Slc25a30

42 Srsf11

43 Stard3nl

$44 \quad$ Strn3

45 Tmem39a

46 Upk1b

$47 \quad$ AI314180

$48 \quad$ Ccdc66

49 Chst13

50 Crls 1

51 Ddit3

52 Gpc6

53 Hrsp12

54 Ikzf2

55 Mbnl1

$56 \quad$ Nit2

$57 \quad \mathrm{Npr} 3$

$58 \quad \mathrm{Pja} 2$

59 S100pbp

60 Siglech

$61 \quad$ Snx27

62 Stk3

63 Taok1

64 Tgfbr3

65 Tpbg

66 Trak2

67 Ttc5

68 Whsc111

69 Zeb2

70 Atrx

71 Copa

72 Cux 1

73 Dmtf1

74 Dnajb6

75
$146 \mathrm{Hmgcr}$

147 Igf1r

$148 \quad$ Klhl28

149 Mboat7

150 Pddc1

151 Ppp1r10

152 Prpf39

153 Pura

154 Rbm25

155 Rnd3

156 Slc7a11

157 Trps1

158 Ttc 14

$160 \quad$ Vps13a

161 Yipf4

162 Add3

163 Atxn713b

164 BC052688

$\begin{array}{llll}164 & \text { BC052688 } & 293 & \text { Dusp3 } \\ 165 & \text { C230076A16Rik } & 294 & \text { Klhl24 }\end{array}$

166 Col8a1

167 Cpeb2

168 Cpne3

169 Dab2

170 Hpgds

171 Lrrc41

172 Lrrk1

173 Nrk

$174 \mathrm{Pbx} 1$

175 Prkx

176 Rab23

177 Rbms2

178 Sec61a2

179 Srgap2

180 Steap4

181 Taf15

182 Tmtc4

183 Usp9x

184 Wac

185 Actr2

186 Cdk6

187 Cflar

188 Ddi2

189 Dnajc13

190 Dyrk2

191 Etnk1

192 Gadd45a

193 Khsrp

194 Mpdz

195 N4bp212

196 Otub1

197 Rab31

198 Sorbs1

199 Tacc1

200 Tmem80

201 Tnrc6a

202 Wnt16

203 Zcchc7

204 Zdhhc2

E130308A19Rik 205 Zfand5

305 Prkacb
159 Vma21
276 Dtx31

277 Hsph1

278 Lin7c

279 Peg3

280 Ppp4c

281 Pum2

282 Rad51c

283 Scd 2

284 Slc12a2

285 Sp7

286 Stk25

287 Txnip

288 Usp48

289 Zfp825

290 AA409587

291 B4galt6

292 D9Ertd292e

295 Msn

296 Ppp3cb

297 Smad5

298 Canx

299 Ccl2

$300 \quad \mathrm{Ccl} 7$

301 Dnm11

302 Fbn2

303 Jmjd1c

304 Nlgn 3

306 Selk

307 Smurf2

308 Srsf1

309 Ssr1

310 Cd209a

311 Dhdh

312 Fn 1

313 Hacd3

314 Nbeal1

315 Ncaph2

316 Ss 18

317 Zfp266

318 Zfp3611

319 4930447C04Rik

320 Adam 10

321 Arpc4

322 Atp8a1

323 Ddx6

324 Malat1

325 Nid 1

326 Snhg11

327 Cx3cr1

328 Dlk1

329 Etv5

330 Mier1

331 Rsrc2

332 Sdf211

333 Slc9a2

334 Atic
Table SII. Continued.

\begin{tabular}{cccccc}
\hline Gene & Gene & Gene & Gene & Gene & Gene \\
count & symbol & count & symbol & count & symbol
\end{tabular}

\begin{tabular}{|c|c|c|c|c|c|}
\hline 76 & Epha7 & 206 & Zfp871 & 335 & Camk1d \\
\hline 77 & Fam49a & 207 & Araf & 336 & $\mathrm{Gm} 2 \mathrm{a}$ \\
\hline 78 & Gcc2 & 208 & Brd4 & 337 & Mirg \\
\hline 79 & Glg1 & 209 & Cwf1912 & 338 & Mob1a \\
\hline 80 & Kdelr2 & 210 & Decr2 & 339 & Peli2 \\
\hline 81 & Lnpep & 211 & Hoxb5os & 340 & Pknox1 \\
\hline 82 & Mcf2 & 212 & Hsd17b12 & 341 & Thbs1 \\
\hline 83 & Pcdhb15 & 213 & Krit1 & 342 & Trove2 \\
\hline 84 & Pum1 & 214 & Larp4 & 343 & Dyrk1a \\
\hline 85 & Rhou & 215 & Mapre1 & 344 & Efnb2 \\
\hline 86 & Ssbp2 & 216 & Phip & 345 & Fgfr2 \\
\hline 87 & Stoml2 & 217 & Rbms3 & 346 & $\mathrm{Gmfb}$ \\
\hline 88 & Syne2 & 218 & Rhobtb3 & 347 & Lcor \\
\hline 89 & Sypl & 219 & Tspyl1 & 348 & Slc16a6 \\
\hline 90 & Tet1 & 220 & Usf1 & 349 & Sox6 \\
\hline 91 & Tfpi & 221 & Apool & 350 & Srrm2 \\
\hline 92 & Tmed7 & 222 & Ascc3 & 351 & Ugt8a \\
\hline 93 & $\operatorname{Trp53}$ & 223 & Cask & 352 & Arhgef11 \\
\hline 94 & Usp4 & 224 & $\mathrm{Cd} 247$ & 353 & Braf \\
\hline 95 & Ywhaz & 225 & Cdh11 & 354 & Dher24 \\
\hline 96 & Zbtb20 & 226 & Cdkn2c & 355 & Golt1b \\
\hline 97 & Zcchc3 & 227 & Cpxm1 & 356 & Otud4 \\
\hline 98 & 1810014B01Rik & 228 & Cyp20a1 & 357 & $\operatorname{Pfdn} 2$ \\
\hline 99 & Adamts 15 & 229 & Erce4 & 358 & $\mathrm{Saa} 2$ \\
\hline 100 & Aplnr & 230 & Fbx13 & 359 & Smg1 \\
\hline 101 & Arl8a & 231 & Ggact & 360 & Igf2 \\
\hline 102 & Btbd7 & 232 & Hnrnpl & 361 & Lsm12 \\
\hline 103 & Crim1 & 233 & Hook3 & 362 & Malt1 \\
\hline 104 & Fubp1 & 234 & Kantr & 363 & Sc5d \\
\hline 105 & Kans11 & 235 & Msi2 & 364 & Tial1 \\
\hline 106 & LOC552902 & 236 & Rab14 & 365 & Zfp451 \\
\hline 107 & Lpp & 237 & Rbm12b1 & 366 & Cxadr \\
\hline 108 & Mau2 & 238 & Smek1 & 367 & 9430020K01Rik \\
\hline 109 & Mmgt1 & 239 & Vtila & 368 & $\mathrm{Gpc} 3$ \\
\hline 110 & Mtpn & 240 & 1300017J02Rik & 369 & Serinc3 \\
\hline 111 & Ntrk2 & 241 & E030016H06Rik & 370 & E230029C05Rik \\
\hline 112 & Nudcd1 & 242 & Eif4ebp2 & 371 & Rbm5 \\
\hline 113 & Peak1 & 243 & Gid4 & 372 & Tgfbr1 \\
\hline 114 & Phf14 & 244 & Hsd17b7 & 373 & Yes1 \\
\hline 115 & Polh & 245 & Kat2b & 374 & Vcan \\
\hline 116 & Ppm1k & 246 & Kif $1 b$ & 375 & Irf2bp2 \\
\hline 117 & Psmb2 & 247 & Map3k2 & 376 & Dapl1 \\
\hline 118 & Rrm2b & 248 & Nek4 & 377 & Pdia4 \\
\hline 119 & Sfpq & 249 & Ppig & 378 & Uba6 \\
\hline 120 & Sgcd & 250 & Rbm41 & 379 & Wif1 \\
\hline 121 & Sp3 & 251 & Tmem45a & 380 & Slc22a8 \\
\hline 122 & Srpx & 252 & Vamp3 & 381 & Ubfd1 \\
\hline 123 & Stxbp4 & 253 & March7 & 382 & Eya1 \\
\hline 124 & Tcf712 & 254 & 9430053009Rik & 383 & Slc38a5 \\
\hline 125 & Vps13b & 255 & Abi2 & 384 & Nnat \\
\hline 126 & Wnt5a & 256 & Arf6 & 385 & Asgr1 \\
\hline 127 & Zcchc24 & 257 & Arglu1 & 386 & Ranbp31 \\
\hline 128 & Zfp207 & 258 & Cspg5 & 387 & Sall1 \\
\hline 129 & Zkscan8 & 259 & Cybb & 388 & Fabp7 \\
\hline 130 & 5830407P18Rik & & & & \\
\hline
\end{tabular}

recent decades, distinct molecular functions and morphometric abnormalities between DPN in T1- and T2DM have been reported $(7,8)$. In addition, it has been proposed that the therapeutic interventions for DPN should differ based on the type of diabetes (7). For DPN in T1DM, it has been suggested that improved glycemic control may preserve nerve function and/or 
Table SIII. List of genes specific to type 2 diabetes mellitus.

\begin{tabular}{lccccc}
$\begin{array}{l}\text { Gene } \\
\text { count }\end{array}$ & $\begin{array}{c}\text { Gene } \\
\text { symbol }\end{array}$ & $\begin{array}{c}\text { Gene } \\
\text { count }\end{array}$ & $\begin{array}{c}\text { Gene } \\
\text { symbol }\end{array}$ & $\begin{array}{c}\text { Gene } \\
\text { count }\end{array}$ & $\begin{array}{c}\text { Gene } \\
\text { symbol }\end{array}$ \\
\hline
\end{tabular}

\section{Upregulated}

1 Sept2

2 Tph2

3 Lipf

4 Ighm

5 Trdn

$6 \operatorname{Prr} 32$

7 Oxtr

$\begin{array}{ll}8 & \text { Cpa2 } \\ 9 & \text { Gpr50 }\end{array}$

$10 \mathrm{Mbnl} 3$

11 Chil3

12 Ucp1

13 Ubd

14 Atp1a4

15 Ighg

16 Dusp9

$17 \quad$ Cdk14

18 Hmga2

19 Atp6v0d2

20 Tfr2

Gabrr2

$\begin{array}{ll}242 & \text { Alcam } \\ 243 & \text { Duoxa1 } \\ 244 & \text { Lrrc3 } \\ 245 & \text { Tcerg11 } \\ 246 & \text { Irx3 } \\ 247 & \text { Lep } \\ 248 & \text { Slc16a10 } \\ 249 & \text { Clspn } \\ 250 & \text { Ctcflos } \\ 251 & \text { Uhrf1 } \\ 252 & \text { Cc12 }\end{array}$

48 Bex 1

484 Cables 1

$485 \quad \mathrm{Ccl} 4$

486 Cnr2

487 Epgn

488 Lpcat3

489 Nabp1

$490 \quad$ Slc24a3

491 Tnfrsf23

492 Bcl2113

$493 \mathrm{Cd} 320$

253 Stc2

254 Gm6484

255 Trav9d-3

256 Gm7694

257 Iqgap3

258 Otop1

259 Ocstamp

260 Mylk

261 Ube2t

2010309G21Rik 263 Dhx32

Mmp12 264 Cd200r1

I11rn $265 \quad \mathrm{Ccl} 22$

9030619P08Rik 266 Ghrh

507 Hoxc13

$\begin{array}{lllll}\text { Arg1 } & 267 & \text { A530020G20Rik } & 508 & \text { Krt222 }\end{array}$

Olr1 268 A530053G22Rik 509 Pfkl

$\begin{array}{lllll}\text { Slc5a7 } & 269 & \text { Aifm2 } & 510 & \text { Pik3ap1 }\end{array}$

Ptchd4

270 4933427G17Rik 511 Sh3tc1

F7

I17r

Apoc4

271 Lat2

272 Prkag3

Spc25

273 Ddias

274 Dgat2

Mogat2

275 Trim67

Lppr4

Crisp2

276 Cenpe

Npr3

277 Cd16412

Rnase2a

278 Mest

Rgs 1

279 Mgll

4930502E18Rik 281 Gdf3

Klf14 282 Vsig4

Crtac1 283 Adora1

Synpo2

284 Il13ra2

Itgax

285 Tmem37

Sez61

286 Cd1d1

Cdca71

287 Kif2c

Serpine1

288 Rrm2

Hmmr

289 Cks2

290 Pdcd1lg2

Fam110c

291 Ffar2

Kenj14

292 Stap1

Prr16

Cldn23

293 Pm20d1

Cyp2r1

294 Clec $4 \mathrm{a} 2$

295 Fmr1nb

$\mathrm{Mc} 2 \mathrm{r}$

296 Blnk

Grtp1

297 Tnfrsf12a

4930480G23Rik 298 Vegfc

Dcst1 299 Acaalb

Lpgat1 $300 \quad$ Als2cr12

Aadac

301 Car9

61 2310002L09Rik 302 Gpt

62 Cyp2c70 303 Havcr2

63 Nek2

64 Dnajb13

65 Gsdma2

304 Marco

305 4931406C07Rik

306 Dnmt3a

502 Trim 25

503 Wfdc21

505 Lman1
Table SIII. Continued.

Gene Gene Gene Gene Gene Gene

count symbol count symbol count symbol

\begin{tabular}{llllll}
\hline 66 & Zranb3 & 307 & Pik3r5 & 548 & Comt \\
67 & Orm2 & 308 & Abcc3 & 549 & Fsd2 \\
68 & Krt79 & 309 & Fbp2 & 550 & Itgb1 \\
69 & Cxc12 & 310 & Gm15498 & 551 & Plk4 \\
70 & Cdca5 & 311 & Kif1b & 552 & Spc24 \\
71 & Adam8 & 312 & Lpxn & 553 & Tns1 \\
72 & Tcf15 & 313 & Pmepa1 & 554 & Atp2a3 \\
73 & Bub1 & 314 & Slc16a12 & 555 & Myo1f \\
74 & Agtr2 & 315 & Stil & 556 & Pla2g12a \\
75 & Dcstamp & 316 & Nup210 & 557 & 1700017 B05Rik \\
76 & My14 & 317 & Gdf5 & 558 & Pou2af1 \\
77 & C430042M11Rik 318 & Serpina1b & 559 & Rpl391 \\
78 & Stra61 & 319 & Bche & 560 & A930018P22Rik \\
79 & Tshr & 320 & Ncaph & 561 & Gm15972 \\
80 & S100a8 & 321 & Stom & 562 & Usp29
\end{tabular}

$80 \quad$ S100a8

81 Ncan

82 S100g

83 Kenj15

84 Pkp2

85 1600025M17Rik

321 Stom

322 Hist $1 \mathrm{~h} 3 \mathrm{~d}$

562 Usp29

563 Arl4a

$\begin{array}{llll}323 & \text { Tmem178 } & 564 & \text { Ercc61 } \\ 324 & \text { 2610020C07Rik } & 565 & \text { Ccl7 }\end{array}$

325 Rps6ka3 566 Shmt1

504 Hist1h1e

\section{8}

\section{2}

93

95
96

$$
97
$$

(1)

100

100

102

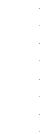

(10)

107

(1)

(111

11

113

116

116

1

12

1

12

(2)

128

86 Gas 212

87

\section{9}

\section{4}

104

$\begin{array}{lll}\text { Gas212 } & 327 & \text { Trabd2b } \\ \text { D830036C21Rik } & 328 & \text { Asf1b }\end{array}$

\section{D830036C21Rik 328 Asf1b}

Slc37a2 329 Edem

Ccnb2 $330 \quad$ Cdt1

Angptl3

331 Cenpa

332 AF251705

Gpnmb

Cldn 15

Cep55

Abcb4

Gprin3

Car6

Brca1

9 Igkv15-103

333 Hipk1

334 Hn11

335 Nepn

336 Sel11

337 Cyp4b1

338 Muc13

339 Rhof

340 Mcm10

341 Tnfrsf11a

342 Ppih

344 Srp54b

345 Gzme

346 Fam126b

347 Heph

348 Cd200r4

349 Cnnm2

108 Dusp3

109 Tmem182

110 Acoxl

111 Chrna2

112 Apoc2

113 Fzd4

114 Fads3

115 Arhgap25

$350 \mathrm{Ghr}$

351 Cenpw

352 Mcm5

353 Ska3

354 Mrgprg

$355 \quad$ Pcdh7

356 2810408I11Rik

357 E2f8

358 5430437J10Rik

117 Ccnb1

118 Gm6277

359 Kif18b

19 4930486L24Rik 360 Akr1c13

120 Hephl1 361 Cd300a

$121 \quad$ Tpx2 $362 \quad$ Cd84

122 Slpi 363 Lrrc27

123 Sirpb1a 364 Mcemp1

24 Tlr13 365 Adam12

125 Saa3 366 Ckap2

126 9130204L05Rik 367 Fam101b

127 Cfi $\quad 368$ Igsf6

128 Rbm28 369 Pramef12

129 Dnmt31 370 Tnfrsf22

130 Sucnr1

131 Atp6v0a1

371 Ttk
343 Rasgrp2

372 Car12

567 Atp10a

568 Glb112

569 Slc2a10

570 Smoc1

571 Zfp106

572 Arfgef2

573 Gcnt1

574 Hesx 1

575 Mrc2

576 Neurl2

577 Plk1

578 Zfp426

579 AI844685

580 Ckap2l

$581 \quad$ Clip 1

582 Dyrk2

583 E2f7

584 Pex16

585 Qpctl

586 Slc22a4

587 Tmem132a

588 Adam 23

589 D17H6S56E-5

590 Ece2

591 Lipa 
Table SIII. Continued.

\begin{tabular}{cccccc}
\hline $\begin{array}{l}\text { Gene } \\
\text { count }\end{array}$ & $\begin{array}{c}\text { Gene } \\
\text { symbol }\end{array}$ & $\begin{array}{c}\text { Gene } \\
\text { count }\end{array}$ & $\begin{array}{c}\text { Gene } \\
\text { symbol }\end{array}$ & $\begin{array}{c}\text { Gene } \\
\text { count }\end{array}$ & $\begin{array}{c}\text { Gene } \\
\text { symbol }\end{array}$
\end{tabular}

\begin{tabular}{|c|c|c|c|c|c|}
\hline 132 & Irak2 & 373 & Depdc1a & 614 & Gatb \\
\hline 133 & Prss27 & 374 & Fam64a & 615 & Gm11827 \\
\hline 134 & Birc5 & 375 & Hpgd & 616 & Ptpn22 \\
\hline 135 & Mis18bp1 & 376 & Dbf4 & 617 & Stx 17 \\
\hline 136 & Spaca1 & 377 & Igsf21 & 618 & Trib3 \\
\hline 137 & Adh6b & 378 & Kcna5 & 619 & E2f2 \\
\hline 138 & Afp & 379 & AI481207 & 620 & Itsn1 \\
\hline 139 & Tk1 & 380 & Cenph & 621 & $\mathrm{C} 2 \mathrm{~cd} 2$ \\
\hline 140 & $\mathrm{Cxcl} 3$ & 381 & Gla & 622 & $\mathrm{Cd} 44$ \\
\hline 141 & Sdsl & 382 & Hrct1 & 623 & $\mathrm{Hfe}$ \\
\hline 142 & $\operatorname{Tsc} 22 \mathrm{~d} 2$ & 383 & Naip1 & 624 & Mmp19 \\
\hline 143 & Klk1 & 384 & Slc18a1 & 625 & 2500002B13Rik \\
\hline 144 & Fosl1 & 385 & 2210406H18Rik & 626 & 2700099C18Rik \\
\hline 145 & Rnf128 & 386 & 3930402G23Rik & 627 & Ano9 \\
\hline 146 & Cdca8 & 387 & Adap2 & 628 & Ccne1 \\
\hline 147 & Timp4 & 388 & Palmd & 629 & Lox \\
\hline 148 & $\mathrm{Zc} 3 \mathrm{~h} 12 \mathrm{~d}$ & 389 & Vldlr & 630 & Dynlrb2 \\
\hline 149 & Plin5 & 390 & Mfge8 & 631 & Eny2 \\
\hline 150 & Ttc39b & 391 & Mpp7 & 632 & Gm3912 \\
\hline 151 & AA467197 & 392 & Sgol1 & 633 & Lipg \\
\hline 152 & Tenm4 & 393 & Mmp13 & 634 & Maff \\
\hline 153 & Nuf2 & 394 & Ms4a6d & 635 & Milr1 \\
\hline 154 & 8430408G22Rik & 395 & Tatdn2 & 636 & Tmem82 \\
\hline 155 & $\mathrm{Cdca} 3$ & 396 & Pigt & 637 & 2600006K01Rik \\
\hline 156 & Hilpda & 397 & Sbk1 & 638 & Acvr1c \\
\hline 157 & Esco2 & 398 & Lrg1 & 639 & $\operatorname{Bcl} 2110$ \\
\hline 158 & Slc9a6 & 399 & Oit3 & 640 & Itih5 \\
\hline 159 & Trem2 & 400 & Qsox1 & 641 & Plekhm1 \\
\hline 160 & Pkp1 & 401 & Vwa8 & 642 & Slc7a8 \\
\hline 161 & Tex33 & 402 & Atp8b4 & 643 & Tes \\
\hline 162 & Aqp7 & 403 & B430306N03Rik & 644 & Cd6 \\
\hline 163 & Fmr1 & 404 & Msr1 & 645 & Gsg2 \\
\hline 164 & Fggy & 405 & $\mathrm{Nfe} 212$ & 646 & Gm2011 \\
\hline 165 & BE949265 & 406 & Nkd1 & 647 & Incenp \\
\hline 166 & Ube2c & 407 & Ptx 3 & 648 & Pold3 \\
\hline 167 & $\mathrm{Pbk}$ & 408 & $\mathrm{Cd} 22$ & 649 & Bst1 \\
\hline 168 & Wif1 & 409 & Galns & 650 & Optc \\
\hline 169 & Nedd9 & 410 & Afmid & 651 & Smad6 \\
\hline 170 & Igfbp3 & 411 & $\mathrm{Cd} 3001 \mathrm{~g}$ & 652 & Tmem206 \\
\hline 171 & Slc7a10 & 412 & Ermp1 & 653 & Zfp787 \\
\hline 172 & Spdl1 & 413 & Fcgr4 & 654 & Cdc20 \\
\hline 173 & Tmed5 & 414 & Shcbp1 & 655 & Ripk3 \\
\hline 174 & Slc15a3 & 415 & Knop1 & 656 & Sdcbp2 \\
\hline 175 & Clec4d & 416 & Serpina12 & 657 & Myo19 \\
\hline 176 & Serpinb9b & 417 & Larp1b & 658 & Tinagl1 \\
\hline 177 & Cux2 & 418 & Thbs 1 & 659 & Tmem106a \\
\hline 178 & Cdk18 & 419 & Seh11 & 660 & Trim16 \\
\hline 179 & C6 & 420 & Lgals3 & 661 & 5033430I15Rik \\
\hline 180 & Lhfpl2 & 421 & Nek6 & 662 & AI606473 \\
\hline 181 & Scel & 422 & Ctse & 663 & Dolk \\
\hline 182 & Glyctk & 423 & Hsd11b2 & 664 & Laptm5 \\
\hline 183 & Mms221 & 424 & Itgb2 & 665 & Lats2 \\
\hline 184 & Tcp11x2 & 425 & Slamf7 & 666 & Lbp \\
\hline 185 & Cacnale & 426 & 3110027N22Rik & 667 & Ormdl3 \\
\hline 186 & Hipk2 & 427 & Dmrt2 & 668 & Pde1b \\
\hline 187 & $\mathrm{Ccl} 3$ & 428 & Hspb6 & 669 & Prelp \\
\hline 188 & Fabp12 & 429 & Ptbp3 & 670 & Serpina $3 c$ \\
\hline 189 & Ncapg & 430 & Slc22a12 & 671 & $\mathrm{Hr}$ \\
\hline 190 & Pnpla2 & 431 & Ttc7 & 672 & K1hl25 \\
\hline 191 & Dppa3 & 432 & Cenpi & 673 & Shb \\
\hline 192 & Elov13 & 433 & Cxc19 & 674 & Vav1 \\
\hline 193 & Prss46 & 434 & Itga7 & 675 & 6030422H21Rik \\
\hline 194 & Mmp8 & 435 & Len2 & 676 & Ccdc86 \\
\hline 195 & Folh1 & 436 & Pid1 & 677 & Cenpp \\
\hline 196 & Cenpf & 437 & Prc1 & 678 & Gch 1 \\
\hline 197 & Npy & 438 & 1700112E06Rik & 679 & Ms4a6b \\
\hline
\end{tabular}

Table SIII. Continued.

Gene Gene Gene Gene Gene Gene

count symbol count symbol count symbol

\begin{tabular}{llllll}
\hline 198 & Apol6 & 439 & 2010003K11Rik & 680 & Nlrc3 \\
199 & Rrbp1 & 440 & Plekhg6 & 681 & Gm9899 \\
200 & Pex51 & 441 & Kif22 & 682 & Phtf2 \\
201 & F10 & 442 & Ndc80 & 683 & Tead4 \\
202 & Ccna2 & 443 & Nlrp10 & 684 & Tfcp211 \\
203 & Tmem135 & 444 & Ptges31 & 685 & Tfpi2 \\
204 & Aspm & 445 & Sod3 & 686 & Map2k5 \\
205 & Gm8350 & 446 & Abhd15 & 687 & Sh2b2 \\
206 & Nus1 & 447 & Acs11 & 688 & Spint2 \\
207 & Tm4sf5 & 448 & Ltc4s & 689 & Wdr62 \\
208 & Cdr2 & 449 & Crk & 690 & 4930511E03Rik
\end{tabular}

209 Aurkb $\quad 450$ Naip2 691 B230307C23Rik

210 Lonrf3 $\quad 451$ Peg3 $\quad 692$ Cib2

211 Bcar3 $452 \quad 4430402$ I18Rik 693 Olfm2

212 Dsg1a $\quad 453$ Fgf21 $\quad 694$ Zfp655

213 Peg10 454 Lrrc28 695 Cenpk

214 Tmem38b 455 Plekhf2 696 Dgkd

215 Clec4e $\quad 456$ Rad51ap1 697 Hspa12a

216 Galnt6 457 Sgol2a $\quad 698$ Hspg2

217 Gys2 $\quad 458$ Ehd2 699 Morn5

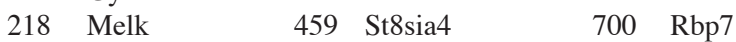

219 Spag5 460 Dcbld1 701 Chpt1

220 Gsdma $\quad 461$ Mtor $\quad 702$ Ehd3

221 Glipr1 462 Plaur $\quad 703$ Hk3

222 Pde7a $\quad 463$ Akap2 $\quad 704$ LOC545086

223 Top2a 464 Ccnf $\quad 705$ Mta3

224 Rab38 $\quad 465$ Lrr1 $\quad 706 \quad$ Spry4

225 Adh4 $\quad 466$ Nat1 407 Treml1

226 Cxc110 $\quad 467 \quad$ Gm31718 $\quad 708$ Gpr65

$\begin{array}{llllll}227 & \text { Kif11 } & 468 & \text { Kif20a } & 709 & \text { Rel }\end{array}$

228 Hrasls $\quad 469$ Optn $\quad 710$ Stk17b

229 Kenk3 $\quad 470$ Plod1 $\quad 711 \quad$ Zfp69

$230 \quad 2810417$ H13Rik 471 Rad54b $\quad 712$ Ccr7

231 1700029I15Rik 472 AU021933 713 Cenpn

232 Serpina3a 473 Ccrl2 $\quad 714 \quad$ Fbxo42

233 Lamb3 $\quad 474$ Piezo1 $\quad 715$ Ttpa

234 Plet1 $\quad 475$ Stab2 $\quad 716 \quad$ Vac14

235 Klb $\quad 476$ C5ar1 $\quad 717$ Ncf1

$\begin{array}{llllll}236 & \text { Btc } & 477 & \text { Hgf } & 718 & \text { Ybx2 }\end{array}$

$\begin{array}{llllll}237 & \text { Ccdc125 } & 478 & \text { Kbtbd12 } & 719 & \text { Cd300ld }\end{array}$

238 Rbpms $\quad 479$ Map7 $\quad 720$ Gk

239 Mettl24 $\quad 480 \quad \operatorname{Rad} 18 \quad 721 \quad$ Xrcc3

240 Tnc $\quad 481$ Sdk2

241 Tpen2 482 Tlr8

Downregulated

$\begin{array}{rlllll}1 & \text { March2 } & 366 & \text { Tspan2 } & 731 & \text { St6gal2 } \\ 2 & \text { March4 } & 367 & \text { Ttyh1 } & 732 & \text { Syt12 } \\ 3 & \text { March5 } & 368 & \text { Unc13a } & 733 & \text { Tnfrsf19 } \\ 4 & \text { March6 } & 369 & \text { Zfp612 } & 734 & \text { Agr2 } \\ 5 & \text { 2900055J20Rik } & 370 & \text { 1110002L01Rik } & 735 & \text { Bcan } \\ 6 & \text { Bcl11b } & 371 & \text { 9330184L24Rik } & 736 & \text { C030034E14Rik } \\ 7 & \text { Cdk7 } & 372 & \text { Aak1 } & 737 & \text { Cntn2 } \\ 8 & \text { Esr1 } & 373 & \text { Adamts1 } & 738 & \text { Dcdc2a } \\ 9 & \text { Gm13446 } & 374 & \text { Aif11 } & 739 & \text { E130002L11Rik } \\ 10 & \text { Gpr85 } & 375 & \text { Ank3 } & 740 & \text { Fam131a } \\ 11 & \text { Hoxb9 } & 376 & \text { Atl1 } & 741 & \text { Fam167a } \\ 12 & \text { Htr7 } & 377 & \text { B930095G15Rik } & 742 & \text { Fam169a } \\ 13 & \text { Kctd14 } & 378 & \text { C030039L03Rik } & 743 & \text { Gap43 } \\ 14 & \text { Mapk4 } & 379 & \text { Cacnb3 } & 744 & \text { Gng4 } \\ 15 & \text { Ncdn } & 380 & \text { Calb2 } & 745 & \text { Kcnd1 } \\ 16 & \text { Osbp13 } & 381 & \text { Ccdc136 } & 746 & \text { Krtdap } \\ 17 & \text { Pfn2 } & 382 & \text { Cdh8 } & 747 & \text { Lrrn1 } \\ 18 & \text { Pou3f2 } & 383 & \text { Clasp2 } & 748 & \text { Lysmd4 } \\ 19 & \text { Slc14a1 } & 384 & \text { Dlg4 } & 749 & \text { Mmd2 } \\ 20 & \text { Tardbp } & 385 & \text { Epb4114b } & 750 & \text { Nacad } \\ 21 & \text { 4833423F13Rik } & 386 & \text { Fbxo30 } & 751 & \text { Necab1 }\end{array}$


Table SIII. Continued.

\begin{tabular}{lccccc}
\hline $\begin{array}{l}\text { Gene } \\
\text { count }\end{array}$ & $\begin{array}{c}\text { Gene } \\
\text { symbol }\end{array}$ & $\begin{array}{c}\text { Gene } \\
\text { count }\end{array}$ & $\begin{array}{c}\text { Gene } \\
\text { symbol }\end{array}$ & $\begin{array}{c}\text { Gene } \\
\text { count }\end{array}$ & $\begin{array}{c}\text { Gene } \\
\text { symbol }\end{array}$
\end{tabular}

\begin{tabular}{llllll}
\hline 22 & Angel1 & 387 & Gpr61 & 752 & Nrxn1 \\
23 & BC030500 & 388 & Itgb3bp & 753 & Nxpe3 \\
24 & Cacng4 & 389 & Lcat & 754 & Parm1 \\
25 & Chd9 & 390 & Lect1 & 755 & Ptprt \\
26 & Cst6 & 391 & Magi1 & 756 & Pvalb \\
27 & Dpys13 & 392 & Meg3 & 757 & Rem2 \\
28 & Ece1 & 393 & Mmp9 & 758 & Rps6ka6 \\
29 & Eif5a2 & 394 & Neto2 & 759 & Sphkap \\
30 & Endod1 & 395 & Otud7a & 760 & Syt11 \\
31 & Epha5 & 396 & Phactr1 & 761 & Th \\
32 & Evi5 & 397 & Prkcz & 762 & Thy1 \\
33 & Gjd2 & 398 & Psd2 & 763 & Tmem74
\end{tabular}

Hs6st2

Htr1d

Ica11

Kalrn

Kenmb2

Kenv1

Mag

Nxph1

Osbpl6

Pax3

Pcdh9

Pcp411

Pter

Rgs7

Scmh 1

Slc1a6

Smco3

Sostdc1

Stxbp 1

Stxbp5

Tmem $229 b$

Ube2e3

Upp2

Zfp788

399 Rnf130

400 Sema3b

401 Sema4g

402 Slc2a3

403 Smr2

404 Srr

405 Trpm3

764 Trim37

765 Tspan8

766 Wipf3

767 Bves

768 Ccdc92

769 Elfn1

406 Zfp385b -

407 A830010M20Rik 772 Gca

408 AI661384 773 Ggt7

409 AI854703 774 Grial

410 AW046457 775 Hoxb5

411 Adam22 776 Kcnh1

412 B3gat1 777 Kcnip1

413 Baiap211 778 Kcnmb1

414 C530044C16Rik 779 Lrfn5

415 Chrnb2 $780 \quad$ Mab2112

416 Cntnap5a

417 Cplx2

418 Crh

419 Csdc2

420 Ctxn1

421 D2Ertd282e

422 Dclk1

5330417C22Rik 424 Erich6

A730089K16Rik 425 Gm36529

Abhd10 426 Greb1l

Adgra1

427 Grm4

C79242

Camk4

Cep97

Cpsf2

Cpsf6

Csmd3

Cyfip2

Eml2

Fat3

Fgf18

Golga7b

Gpr173

Grin1

Hs3st1

Irf4

Klf12

Lancl1

Lrba

Lrrn4cl

Lrrtm4

Nppb

Paqr5

Plch1

Prokr2

Prr51
428 Hopx

429 Mycn

430 Nalcn

431 Nebl

432 Nova1

433 Pcp4

434 Plekha6

435 Rab6b

436 Rbfox 3

437 Rbm11

$438 \quad \operatorname{Rgs} 8$

439 Rtn4

440 Sarm1

441 Sema3e

442 Shisa4

$443 \quad$ Slc6a17

444 Slco1c1

445 Synm

446 Tubg2

447 Wdr17

448 9330182L06Rik

449 Aifm3

450 Bhlha9

451 Cd200r3

$452 \mathrm{Cdh} 1$

Table SIII. Continued.

Gene Gene Gene Gene Gene Gene

count symbol count symbol count symbol

\begin{tabular}{|c|c|c|c|c|c|}
\hline 88 & R3hdm1 & 453 & Chd5 & 818 & Nrsn 1 \\
\hline 89 & Runx1t1 & 454 & Dgkg & 819 & Pcsk1 \\
\hline 90 & Sall4 & 455 & E130309D14Rik & 820 & Pcyt1b \\
\hline 91 & Slc29a4 & 456 & Hapln2 & 821 & Rnf157 \\
\hline 92 & Slc35f2 & 457 & Myt11 & 822 & Serpini1 \\
\hline 93 & Trank1 & 458 & Ntsr2 & 823 & Slain1 \\
\hline 94 & Zdhhc18 & 459 & Oprl1 & 824 & Slc35f4 \\
\hline 95 & Zfp318 & 460 & Prdm8 & 825 & Slc4a10 \\
\hline 96 & Zfp37 & 461 & Prr18 & 826 & Vps13c \\
\hline 97 & 2900009J20Rik & 462 & Psmf1 & 827 & Wt1 \\
\hline 98 & A930041H05Rik & 463 & Rab3a & 828 & Adamts 16 \\
\hline 99 & Ak5 & 464 & Rasgef1a & 829 & Ano3 \\
\hline 100 & Arg2 & 465 & Rgmb & 830 & Arfgef3 \\
\hline 101 & Bank1 & 466 & Rgs7bp & 831 & Arhgdig \\
\hline 102 & Ccdc28a & 467 & Robo1 & 832 & Cntn1 \\
\hline 103 & Cdkn1b & 468 & Rph3a & 833 & $\operatorname{Ddx} 25$ \\
\hline 104 & Clec21 & 469 & Slc2a12 & 834 & Dzank1 \\
\hline 105 & Csmd1 & 470 & Slc6a2 & 835 & Fam19a3 \\
\hline 106 & Dip2a & 471 & Sstr4 & 836 & Gprin2 \\
\hline 107 & Dlgap1 & 472 & Syt7 & 837 & Hsbp111 \\
\hline 108 & F5 & 473 & Tdo2 & 838 & Htr4 \\
\hline 109 & Fzd3 & 474 & Tmem108 & 839 & Jakmip1 \\
\hline 110 & Htr1a & 475 & Vwa5a & 840 & Jakmip2 \\
\hline 111 & Hunk & 476 & $\mathrm{Zfr} 2$ & 841 & Klc1 \\
\hline 112 & Klhl32 & 477 & 9530077C05Rik & 842 & Lix 1 \\
\hline 113 & L1cam & 478 & Acs16 & 843 & Maneal \\
\hline 114 & Lrrtm2 & 479 & Aqp4 & 844 & Npy2r \\
\hline 115 & Map6d1 & 480 & Atcay & 845 & Rab3c \\
\hline 116 & Mfsd6 & 481 & Ccdc68 & 846 & Reep2 \\
\hline 117 & Mrpl35 & 482 & Cdk5r1 & 847 & Rufy2 \\
\hline 118 & Nedd4l & 483 & Cgref1 & 848 & $\mathrm{Sh} 3 \mathrm{gl} 2$ \\
\hline 119 & Pcsk6 & 484 & $\mathrm{Cpb} 1$ & 849 & Vstm5 \\
\hline 120 & Plxnb1 & 485 & Ctnnd2 & 850 & 1810041L15Rik \\
\hline 121 & Ppp1r2 & 486 & Cxxc4 & 851 & A830039N20Rik \\
\hline 122 & Rbm33 & 487 & Dnah7a & 852 & Aldoc \\
\hline 123 & $\operatorname{Rf} x 4$ & 488 & Dzip3 & 853 & Asic1 \\
\hline 124 & Sacs & 489 & Fndc4 & 854 & ВС059841 \\
\hline 125 & Serpina11 & 490 & Fxyd7 & 855 & Car10 \\
\hline 126 & Shd & 491 & Gabra5 & 856 & Ccdc 172 \\
\hline 127 & Tcte2 & 492 & Glrb & 857 & Cldn10 \\
\hline 128 & Tia1 & 493 & Lrch1 & 858 & D130043K22Rik \\
\hline 129 & Tox2 & 494 & Mir124a-1hg & 859 & Gabrg2 \\
\hline 130 & Ttbk1 & 495 & Ncam2 & 860 & Gm16551 \\
\hline 131 & Ttc3 & 496 & Plk5 & 861 & Kcnip2 \\
\hline 132 & Vamp4 & 497 & Pogz & 862 & Kcnip4 \\
\hline 133 & 2900056L01Rik & 498 & Prox 1 & 863 & Kif3c \\
\hline 134 & 2900064F13Rik & 499 & Rasl10b & 864 & Mrgpra3 \\
\hline 135 & Arhgap20 & 500 & Rnf208 & 865 & Pappa2 \\
\hline 136 & Ccdc112 & 501 & Scin & 866 & Rab39b \\
\hline 137 & Cfap69 & 502 & Scn8a & 867 & Rassf6 \\
\hline 138 & Cmtm5 & 503 & Scrt1 & 868 & Rbfox 1 \\
\hline 139 & Dtna & 504 & Slitrk4 & 869 & Rufy3 \\
\hline 140 & Dusp15 & 505 & Susd5 & 870 & Sez612 \\
\hline 141 & Ephx4 & 506 & Tmem169 & 871 & Soga3 \\
\hline 142 & Faim2 & 507 & Tmem196 & 872 & Stac \\
\hline 143 & Gabrb3 & 508 & Ttc39c & 873 & $\mathrm{~Sv} 2 \mathrm{a}$ \\
\hline 144 & Galnt14 & 509 & Tyrp1 & 874 & Tmem179 \\
\hline 145 & Gm12371 & 510 & Unc 5c & 875 & Tspyl4 \\
\hline 146 & Gm13889 & 511 & Vwc21 & 876 & Aplp1 \\
\hline 147 & Gprasp1 & 512 & 5930412G12Rik & 877 & Atp6v1g2 \\
\hline 148 & Hspa4l & 513 & Abcg 4 & 878 & Far2 \\
\hline 149 & Igsf11 & 514 & Ache & 879 & Megf11 \\
\hline 150 & Itih3 & 515 & Acsm3 & 880 & Nsg2 \\
\hline 151 & Kcnip3 & 516 & Arhgap12 & 881 & Pclo \\
\hline 152 & Kenk1 & 517 & Cabp7 & 882 & Pou4f2 \\
\hline 153 & Lingo1 & 518 & Ccdc30 & 883 & Ptprn \\
\hline
\end{tabular}


Table SIII. Continued.

\begin{tabular}{lccccc}
\hline $\begin{array}{l}\text { Gene } \\
\text { count }\end{array}$ & $\begin{array}{c}\text { Gene } \\
\text { symbol }\end{array}$ & $\begin{array}{c}\text { Gene } \\
\text { count }\end{array}$ & $\begin{array}{c}\text { Gene } \\
\text { symbol }\end{array}$ & $\begin{array}{c}\text { Gene } \\
\text { count }\end{array}$ & $\begin{array}{c}\text { Gene } \\
\text { symbol }\end{array}$
\end{tabular}

\begin{tabular}{llllll}
\hline 154 & Mkx & 519 & Clip3 & 884 & Rasgrp1 \\
155 & Nipa1 & 520 & Clip4 & 885 & Rit2 \\
156 & Phex & 521 & Dgkb & 886 & Slc1a2 \\
157 & Pnkd & 522 & Diras1 & 887 & Them5 \\
158 & Ppm11 & 523 & Elov12 & 888 & Tmem151b \\
159 & Spink10 & 524 & Gm10700 & 889 & Zwint \\
160 & St3gal6 & 525 & Gpr149 & 890 & $6430604 M 11 R i k$ \\
161 & St6galnac3 & 526 & Has2 & 891 & Bsn \\
162 & Stk33 & 527 & Hoxd10 & 892 & C530008M17Rik \\
163 & Tfap2a & 528 & Hs3st4 & 893 & Cadps2 \\
164 & Wdr31 & 529 & Iqsec3 & 894 & Cbln2 \\
165 & 4930519N06Rik & 530 & Kazn & 895 & Ctnna2 \\
166 & Acsb1 & 531 & Kcnf1 & 896 & Cyp4x1
\end{tabular}

166 Acsbg1 531 Kcnf 1

167 Akr1c18 532 Kif3a

168 Asxl3 533 Lepr

169 Atad1 534 Lingo2

170 B3galt1 535 Mapt

171 B630019K06Rik 536 Mycl

$\begin{array}{llll}172 & \text { Ccdc138 } & 537 & \text { Neto1 }\end{array}$

173 Cdr21 $\quad 538 \quad$ Nipal3

174 Clec3b $\quad 539 \quad$ Nwd2

175 Cyr61 $\quad 540$ Pdzd4

176 Dhh

177 Edil3

178 Fign

179 Gabbr 1

180 Gabra1

181 Gm13629

$182 \quad$ Gm5089

183 Gnaz

184 Gpr62

185 Hen 1

186 Kenk5

187 Kenq5

188 Lrrc49

189 Mbp

190 Pak1

191 Pax9

192 Pcdh10

193 Praf2

194 Prss12

195 Ptchd2

196 Rimklb

197 Sgtb

198 Sh3d19

199 Slc16a9

200 Smpd3

201 Sorcs3

202 Stmn2

203 Usp31

204 Vamp1

205 Vat11

206 Zdbf2

207 Aldh1a2

208 Atp2b4

209 Cacnb4

210 Camk1g

211 Chn1

212 Dio3os

213 Fkbp1b

214 Gjb1

215 Gna14

216 Herc3

217 Hnrnpr

218 Igsf1

219 Irf6

\section{Plcxd3}

542 Plp1

543 Ppfia2

544 Ptbp2

545 Scrn1

546 Slc25a18

547 Slco5a1

548 Svop

549 Syngr 1

550 Tmem246

551 Tmem88b

552 Arpp21

553 Atp1b1

$554 \mathrm{Cd} 209 \mathrm{~g}$

$555 \mathrm{Cda}$

556 Chst5

557 Cnnm1

558 Dleu2

559 Dscam

560 Dusp26

561 Hebp2

562 Il31ra

563 Lhfpl3

564 Lrp8

565 Mfsd4

566 P2rx3

567 Pigz

568 Pnma2

569 Rab39

570 Rell2

571 Rlbp1

572 Sema4f

573 Slitrk5

574 Smarcal1

575 Sntg1

576 Stmn3

577 Susd4

578 Tmem117

896 Сур4x

897 Elavl3

898 Fbxo2

899 Gm10419

900 Igfbp2

$901 \quad$ Jph3

902 Jph4

903 Klhdc8a

904 Nrcam

905 Ppap2c

906 Ppm1j

907 Ptgs2

908 Tcte1

910 Trim9

911 Acpp

912 Adgrb3

913 Anks1b

914 Ccdc184

915 Chga

916 Ckmt1

917 Galnt13

918 Gng3

919 Kcnab1

920 Kenc1

921 Kif5a

$922 \mathrm{Napb}$

923 Nrxn3

924 Ptgds

925 Ptprn2

926 Rgs17

927 Tox3

928 Uchl1

929 Zmat4

930 Csrnp3

931 Dpp6

932 Fam184b

933 Gdap1

934 Kctd8

935 Nrg3

936 Rnf112

937 Syt2

938 Tmem200c

939 3632451O06Rik

940 9430021M05Rik

941 AW060742

942 Ank1

943 Asic3

579 Vwa7

944 Brsk2

580 1700019D03Rik 945 Cldn11

581 A2m

582 Brsk1

946 Faxc

947 Fst15

583 C030011L09Rik 948 Lhfpl5

584 Cabp1
909 Tmem150c
Table SIII. Continued.

Gene Gene Gene Gene Gene Gene

count symbol count symbol count symbol

\begin{tabular}{llllll}
\hline 220 & Ism1 & 585 & Dok4 & 950 & Pacsin1 \\
221 & Jam3 & 586 & Epb4113 & 951 & Rims3 \\
222 & Klf7 & 587 & Exph5 & 952 & Rspo2 \\
223 & Ltbp1 & 588 & Fez1 & 953 & Rundc3a \\
224 & Mapk8ip1 & 589 & Fmn2 & 954 & Scg5 \\
225 & Nek1 & 590 & Gria2 & 955 & Slc6a15 \\
226 & Nrip3 & 591 & Kif5c & 956 & Srrm3 \\
227 & Phyhip & 592 & Mast1 & 957 & St8sia3 \\
228 & Prepl & 593 & Ms4a3 & 958 & Tram111 \\
229 & Rasgrf1 & 594 & Ndrg4 & 959 & Zswim5 \\
230 & Serpind1 & 595 & Pirt & 960 & 2900011O08Rik \\
231 & Sox1 & 596 & Prmt8 & 961 & Astn1 \\
232 & Sox2ot & 597 & Rab33a & 962 & Atp2b2 \\
233 & Spock2 & 598 & Rab37 & 963 & Bend6 \\
234 & Susd2 & 599 & Slc25a12 & 964 & Bex2 \\
235 & Syndig11 & 600 & Srgap3 & 965 & Cacna2d3 \\
236 & Zfhx2 & 601 & Tmem163 & 966 & Cckar \\
237 & A730054J21Rik & 602 & Tmem79 & 967 & Cend1 \\
238 & Adam11 & 603 & Ttc9b & 968 & D930028M14Rik \\
239 & Adcy1 & 604 & $4930524 O 07 R i k$ & 969 & Dnm3 \\
240 & Adgrl3 & 605 & A330050F15Rik & 970 & Fam155a \\
241 & Ankr13d & 606 & A330102110Rik & 971 & Gal
\end{tabular}

241 Ankrd13d 606 A330102I10Rik 971 Gnal

242 Apba1 607 Arhgap28 972 Ipw

243 Apcdd1 608 Arhgap44 973 Kcna4

244 B3gnt5 609 Celf4 974 Kcnj10

245 Bmp7 610 Cntnap4 $\quad 975$ Lsamp

246 Crym $\quad 611$ Elovl7 976 Mapk8ip2

247 Dbndd2 612 Fcrls 977 Mtcl1

248 Eef1a2 $\quad 613 \quad$ Gpr37 $\quad 978$ Phf24

249 Fam81a $\quad 614$ Grin3a $\quad 979$ Snap25

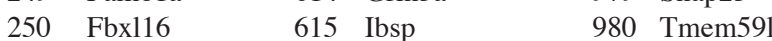

251 Fbxo41 616 Ints6 981 Atp2b3

252 Flrt1 $\quad 617$ Lgi3 $\quad 982$ Col25a1

253 Gkn3 $\quad 618$ Lrrtm1 983 Dpys15

254 Hey2 619 Magee1 984 Grip1

255 Kcnd2 620 Pcdh20 985 Hapln1

256 Keng4 $\quad 621 \quad$ Plcxd2 $\quad 986$ Klk6

257 Lin7a $\quad 622$ Plekhh1 $\quad 987$ Lhfpl4

258 Lrrtm3 $\quad 623$ Prkg2 988 Nap113

259 Ncam1 624 Prune2 989 Ppm1e

260 Nrsn2 $\quad 625$ Rcan2 990 Prph

$\begin{array}{llllll}261 & \text { Pak7 } & 626 & \text { Scn2b } & 991 & \text { Rnf182 }\end{array}$

262 Pcolce2 $\quad 627 \quad$ Slc7a14 $\quad 992$ Rtn1

263 Ppp2r2c $\quad 628$ Stx1b $\quad 993$ Slc39a12

264 Prr15 $\quad 629$ Sult4a1 $\quad 994$ Tmem35

265 S100b $\quad 630$ Syt14 $\quad 995$ Zcchc18

266 Samd14 631 Tmem132e 996 Ano4

267 Sh3gl3 $\quad 632$ Ubash3b $\quad 997$ Arhgef7

268 Slc6a11 633 A830018L16Rik 998 BC048546

269 Trio $\quad 634$ Adcy8 $\quad 999$ Gabrg1

270 2610100L16Rik 635 Cdh10 1000 Hecw1

271 AI846148 636 Ceacam10 1001 Insm2

272 Apbb1 637 Chrna6 1002 Map7d2

273 Ccl27a $\quad 638$ Eno2 1003 Mog

274 Ccp110 639 Fgf4 1004 Pcsk2

275 Cd24a $\quad 640$ Frrs11 1005 Pgbd5

276 Clvs2 641 Gm5124

277 Cntn6 642 Gpr22

$278 \quad$ Cxcl13 643 Il1r2

279 Cytl1 644 Lancl3

280 Depdc5 645 Ogfrl1

281 Dkk2 646 Olfm3

282 Gabrb2 647 Phactr3

283 Gm15663 648 Reep1

284 Hs3st2 $\quad 649$ Rgs4

285 Id4 650 Rimbp2
1006 Rprm

1007 Sgpp2

1008 Slc17a7

1010 Spock 1

1011 Adrb3

1012 Akap6

1013 C030017B01Rik

1014 Calb1

1015 Cpne4
1009 Slc26a7 
Table SIII. Continued.

\begin{tabular}{|c|c|c|c|c|c|c|c|c|c|c|c|}
\hline $\begin{array}{l}\text { Gene } \\
\text { count }\end{array}$ & $\begin{array}{c}\text { Gene } \\
\text { symbol }\end{array}$ & $\begin{array}{l}\text { Gene } \\
\text { count }\end{array}$ & $\begin{array}{c}\text { Gene } \\
\text { symbol }\end{array}$ & $\begin{array}{l}\text { Gene } \\
\text { count }\end{array}$ & $\begin{array}{l}\text { Gene } \\
\text { symbol }\end{array}$ & $\begin{array}{l}\text { Gene } \\
\text { count }\end{array}$ & $\begin{array}{c}\text { Gene } \\
\text { symbol }\end{array}$ & $\begin{array}{l}\text { Gene } \\
\text { count }\end{array}$ & $\begin{array}{l}\text { Gene } \\
\text { symbol }\end{array}$ & $\begin{array}{l}\text { Gene } \\
\text { count }\end{array}$ & $\begin{array}{c}\text { Gene } \\
\text { symbol }\end{array}$ \\
\hline 286 & Il18 & 651 & Skida1 & 1016 & Dync1i1 & 352 & Pip5k11 & 717 & Arhgap36 & 1082 & Prrxl1 \\
\hline 287 & Map1a & 652 & Slc6a1 & 1017 & Isl1 & 353 & $\mathrm{Pla} 2 \mathrm{~g} 2 \mathrm{~d}$ & 718 & Caln 1 & 1083 & AI593442 \\
\hline 288 & Mllt11 & 653 & Slitrk1 & 1018 & Isl2 & 354 & Ppl & 719 & Celf6 & 1084 & Calcb \\
\hline 289 & Nfasc & 654 & Tmem56 & 1019 & Kndc1 & 355 & Prokr1 & 720 & Cntn4 & 1085 & Shh \\
\hline 290 & Pak3 & 655 & Trnp1 & 1020 & Map4 & 356 & Rab27b & 721 & Corin & 1086 & Tmem130 \\
\hline 291 & $\operatorname{Pgm} 211$ & 656 & Tspyl5 & 1021 & Mapk10 & 357 & Rragd & 722 & Epb41 & 1087 & Cyp2f2 \\
\hline 292 & Plcd4 & 657 & Vstm2a & 1022 & Phyhipl & 358 & Rsrp1 & 723 & Fosb & 1088 & Snap91 \\
\hline 293 & Rasal2 & 658 & 4832406H04Rik & 1023 & Pou4f1 & 359 & Scrt2 & 724 & Kcnj3 & 1089 & Nap112 \\
\hline 294 & Rltpr & 659 & A330068G13Rik & 1024 & Rims1 & 360 & Slc $35 \mathrm{~d} 3$ & 725 & Kcnk10 & 1090 & Scn11a \\
\hline 295 & Sec62 & 660 & Aard & 1025 & Sv2b & 361 & Spink2 & 726 & Lrrc75b & 1091 & Myt1 \\
\hline 296 & Snph & 661 & Acsl3 & 1026 & Syt1 & 362 & Stk39 & 727 & Morn4 & 1092 & Grm7 \\
\hline 297 & Stk32c & 662 & Atp8a2 & 1027 & Syt4 & 363 & Synrg & 728 & Onecut2 & 1093 & Tubb3 \\
\hline 298 & Syngr3 & 663 & Cacna1b & 1028 & Tubb4a & 364 & Tmem255a & 729 & Pcsk1n & 1094 & Cadps \\
\hline 299 & Tbc1d 30 & 664 & Cdh9 & 1029 & Amph & 365 & Tshz2 & 730 & $\operatorname{Scg} 3$ & & \\
\hline
\end{tabular}

300 Trim2 665 Ces1f

301 1110032F04Rik 666 Col4a6

302 Ank2 667 Dner

303 Apc2 668 Fn3k

304 Apoh

305 Asphd1

306 BB319198

$669 \mathrm{Gm} 16532$

670 Itga8

671 Kif1a

307 Cacna2d2

308 Cadm3

$309 \quad \operatorname{Clgn}$

310 Cmah

311 Ddn

312 Dhrs2

313 Efcab1

314 Fam189a1

$315 \quad \mathrm{Gm} 32444$

316 Gpr158

317 Gpr75

318 Grm8

319 Inadl

320 Kenq2

321 Kctd4

322 Kif21a

323 Krt27

$324 \quad$ Lgi2

325 Lonrf2

326 Lztfl 1

$327 \mathrm{Mfsd} 2 \mathrm{a}$

328 Mro

329 Penxl2

$330 \quad$ Prkg1

331 Srd5a1

332 Srsf12

333 Tmem231

334 Tnik

335 Trim 36

336 Ttbk2

337 Aox3

338 Ccdc47

339 Celsr2

340 Cldn 9

341 Dbndd1

342 Esd

343 Fxyd3

344 Greb1

345 Gria4

346 Kenn2

347 Kent 1

348 Lynx 1

349 Ndp

$350 \quad$ Nkain2

$351 \quad$ Pi16
672 L1td 1

673 Megf10

674 Mtus2

675 Nipal2

676 Nrxn2

677 Ntrk1

678 Pcdh8

$679 \quad \mathrm{Pla} 2 \mathrm{~g} 3$

680 Rab9b

681 Sowahb

$682 \operatorname{Trpc} 3$

683 Trpc6

684 Tvp23a

685 Vwa5b2

686 A730017C20Rik

687 Amer3

688 Arhgef4

689 Arnt2

690 Ccser 1

691 Cntnap2

692 Cplx 1

694 Dock3

695 Dpp4

696 Fam189b

697 Gdap111

698 Gm2115

699 Gprasp2

700 Hoxb6

701 Lgil

702 Mobp

703 Mogat1

704 Mtmr7

705 Nkx2-2

706 Omg

707 Pde1c

708 Ptprz1

709 Scn1a

710 Slc $35 \mathrm{f} 3$

711 Spire2

712 Stxbp51

713 Tenm1

714 Timd4

715 6330563C09Rik

716 Ap3b2
693 Crygs
Table SIII. Continued.

1030 Elavl4

1031 Ermn

1032 Gfap

1033 Hoxd

1034 Mlc1

1035 Mup10

$1036 \mathrm{Nrg} 1$

1037 Scn10a

1038 Tagln3

1039 Vgf

1040 Add2

1041 Cdhr1

1042 Chrna7

1043 Cpne6

1044 Dlg2

1045 Dpp10

1046 Fam19a4

1047 Grik1

1048 Htr3a

1049 Plekhd1

1050 Ppp1r1c

1051 Ppp2r2b

1052 Ralyl

1053 St8sia1

1054 Stmn4

1055 Synpr

1056 2900052N01Rik

1057 Chgb

1058 Fam19a2

1059 Resp18

1060 Slc17a6

1061 Spock3

1062 Vsnl1

1063 Nefl

1064 Scn9a

$1065 \mathrm{Nts}$

1066 Sult1e1

1067 Avil

1068 2810037O22Rik

1069 Nefh

1070 Cdk5r2

1071 Ttc9

1072 Adcyap1

1073 Pon1

1074 Slc24a2

1075 Nefm

1076 Panx2

1077 Syt9

1078 Rimkla

1079 Gm2102

$1080 \quad$ Scg2

1081 Tac1 decrease the likelihood of developing DPN (6). By contrast, for DPN in T2DM, not only glycemic control, but treatments for other risk factors including obesity may be equally critical in preventing DPN (6). Therefore, since the molecular mechanisms underlying the pathogenesis of DPN in each type of DM may differ, understanding the mechanisms may lead to novel

In the present study, there were marked differences between the gene expression profiles, GO terms and KEGG pathways of DEGs in the sciatic nerves of T1- and T2DM mouse models. This was in accordance with previous studies revealing considerable alterations in global gene expression profiles of several tissues including skeletal muscles and adipose tissue (21), and organs such as the intestine (22), liver (23) and brain (24) in the two types of DM. It is established that T1DM is characterized by destruction of pancreatic islet cells by autoimmunity, with loss of pancreatic insulin production, while T2DM is a metabolic disease with high pancreatic insulin production in the setting of insulin resistance (6). Therefore, the different insulin level and insulin signaling pathways between the two types of DM may converge on and modulate the transcription of genes (25); it thus seemed reasonable to search for altered gene expression patterns in DPN between T1- and T2DM models characterized by absolute or relative insulin deficiency.

A number of DEGs unique to the sciatic nerve of T1DM mice were associated with cell proliferation, as well as the mitogen-activated protein kinase signaling pathway, which is a critical pathway for cell proliferation (26). In neuropathic pain, a correlation has been determined between the proliferation of nerve cells including microglia and astrocyte and the abnormal pain responses $(27,28)$. In addition, previous study has demonstrated that the proliferation of glia cells including astrocyte was associated with structural changes in the nervous system, such as axonal growth (29). Furthermore, with loss of C-fibers, increased frequencies of denervated Schwann cells and regenerating fibers have been identified in T1DM mice but not in T2DM mice (8). Therefore, the DEGs associated with cell proliferation may serve a crucial role during the pathological changes of DPN in T1DM.

Insulin resistance is among the major factors that leads to the development and progression of complications in T2DM (30). therapeutic approaches for prevention or treatment. 
Table I. Top 20 coincident enriched GO terms in T1- and T2DM.

\begin{tabular}{|c|c|c|c|c|c|}
\hline \multirow[b]{2}{*}{ GO ID } & \multirow[b]{2}{*}{ GO term } & \multicolumn{2}{|c|}{ T1DM } & \multicolumn{2}{|c|}{$\mathrm{T} 2 \mathrm{DM}$} \\
\hline & & Gene count & P-value & Gene count & P-value \\
\hline GO:0005515 MF & Protein binding & 57 & $3.1 \mathrm{E}-6$ & 76 & $9.2 \mathrm{E}-20$ \\
\hline GO:0005737 CC & Cytoplasm & 21 & $6.7 \mathrm{E}-6$ & 123 & $3.8 \mathrm{E}-14$ \\
\hline GO:0001525 BP & Angiogenesis & 14 & $9.6 \mathrm{E}-6$ & 89 & 7.3E-18 \\
\hline GO:0000166 MF & Nucleotide binding & 45 & $2.4 \mathrm{E}-5$ & 46 & $5.5 \mathrm{E}-10$ \\
\hline GO:0007399 BP & Nervous system development & 21 & $3.2 \mathrm{E}-5$ & 86 & 7.3E-11 \\
\hline GO:0008201 MF & Heparin binding & 13 & $4.6 \mathrm{E}-5$ & 57 & $5.9 \mathrm{E}-14$ \\
\hline GO:0006468 BP & Protein phosphorylation & 11 & $5.4 \mathrm{E}-5$ & 61 & $4.3 \mathrm{E}-12$ \\
\hline GO:0005578 CC & Proteinaceous extracellular matrix & 24 & $5.5 \mathrm{E}-5$ & 66 & $9.1 \mathrm{E}-20$ \\
\hline GO:0016310 BP & Phosphorylation & 13 & $6.2 \mathrm{E}-5$ & 78 & $8.8 \mathrm{E}-16$ \\
\hline GO:0009986 CC & Cell surface & 12 & $6.5 \mathrm{E}-5$ & 54 & $5.6 \mathrm{E}-17$ \\
\hline GO:0016020 CC & Membrane & 17 & $8.9 \mathrm{E}-5$ & 23 & $3.2 \mathrm{E}-15$ \\
\hline GO:0005794 CC & Golgi apparatus & 12 & $2.4 \mathrm{E}-4$ & 45 & $2.5 \mathrm{E}-17$ \\
\hline GO:0004672 MF & Protein kinase activity & 14 & $3.1 \mathrm{E}-4$ & 24 & 4.2E-16 \\
\hline GO:0030335 BP & Positive regulation of cell migration & 15 & $4.5 \mathrm{E}-4$ & 19 & $8.5 \mathrm{E}-17$ \\
\hline GO:0005783 CC & Endoplasmic reticulum & 6 & 5.3E-4 & 32 & $5.2 \mathrm{E}-16$ \\
\hline GO:0019933 BP & cAMP-mediated signaling & 18 & $5.8 \mathrm{E}-4$ & 30 & $7.5 \mathrm{E}-13$ \\
\hline GO:0048471 CC & Perinuclear region of cytoplasm & 16 & $6.3 \mathrm{E}-4$ & 21 & $5.2 \mathrm{E}-12$ \\
\hline GO:0016301 MF & Kinase activity & 19 & $6.7 \mathrm{E}-4$ & 26 & 7.1E-15 \\
\hline GO:0004674 MF & Protein serine/threonine kinase activity & 32 & $1.3 \mathrm{E}-3$ & 24 & $2.1 \mathrm{E}-18$ \\
\hline GO:0006629 BP & Lipid metabolic process & 22 & $1.6 \mathrm{E}-3$ & 42 & $7.6 \mathrm{E}-22$ \\
\hline
\end{tabular}

GO, Gene ontology; BP, biological process; MF, molecular function; CC, cellular component; T1DM, type 1 diabetes mellitus; T2DM, type 2 diabetes mellitus .

A

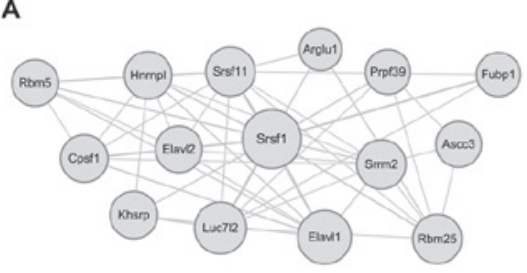

C

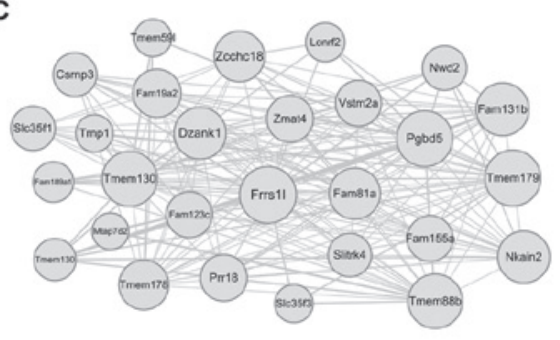

B

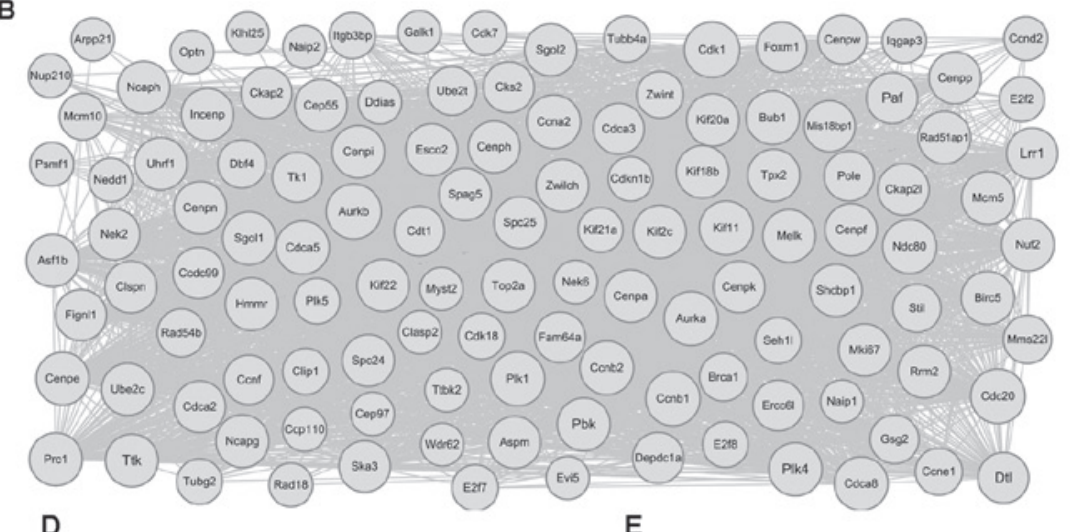

D

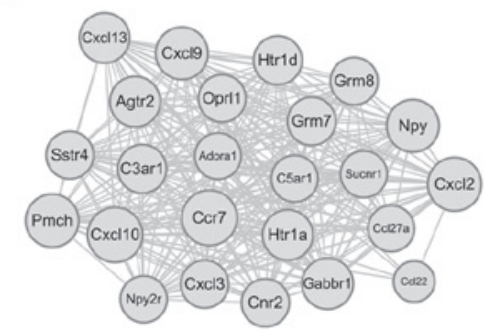

E

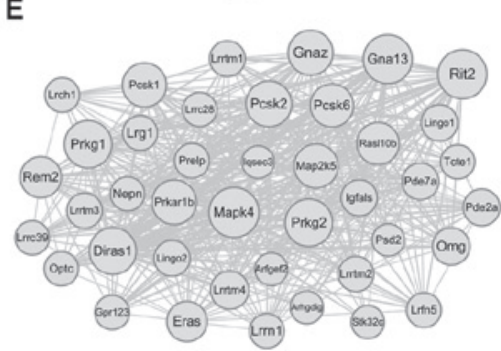

$\mathbf{F}$

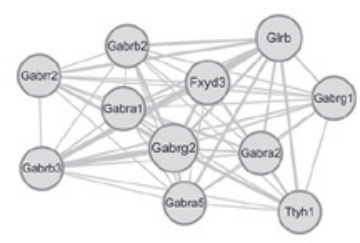

G

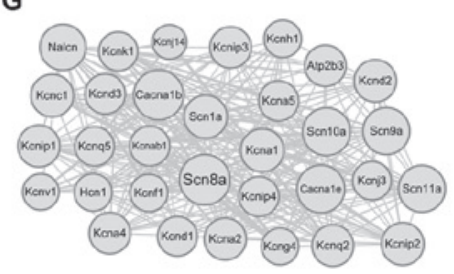

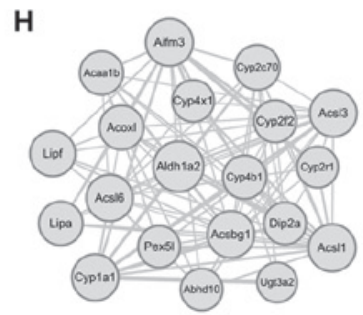

Figure 2. Protein modules in the PPI networks for T1- and T2DM. The size of a protein was determined by the degree of its connection to other proteins, and the width of the edge connecting two proteins by the combined score of those proteins. (A) Protein module in the PPI network for T1DM; (B-H) the 7 modules in the PPI network for T2DM. PPI, protein-protein interaction; T1DM, type 1 diabetes mellitus; T2DM, type 2 diabetes mellitus. 
Table II. Top 20 enriched GO terms for differentially expressed genes in the sciatic nerve of T1- and T2DM models, respectively.

\begin{tabular}{|c|c|c|c|}
\hline GO ID & GO term & Gene count & P-value \\
\hline \multicolumn{4}{|l|}{ T1DM } \\
\hline GO:0005654 CC & Nucleoplasm & 92 & $5.3 \mathrm{E}-7$ \\
\hline GO:0070062 CC & Extracellular exosome & 116 & $1.3 \mathrm{E}-6$ \\
\hline GO:0005829 CC & Cytosol & 84 & $2.8 \mathrm{E}-6$ \\
\hline GO:0045893 BP & Positive regulation of transcription, DNA-templated & 38 & 4.3E-6 \\
\hline GO:0050680 BP & Negative regulation of epithelial cell proliferation & 12 & $6.0 \mathrm{E}-6$ \\
\hline GO:0071560 BP & Cellular response to transforming growth factor $\beta$ stimulus & 11 & $8.3 \mathrm{E}-6$ \\
\hline GO:0005604 CC & Basement membrane & 13 & $1.7 \mathrm{E}-5$ \\
\hline GO:0090090 BP & Negative regulation of canonical Wnt signaling pathway & 13 & $3.5 \mathrm{E}-5$ \\
\hline GO:0045165 BP & Cell fate commitment & 11 & $3.7 \mathrm{E}-5$ \\
\hline GO:0005925 CC & Focal adhesion & 27 & 4.7E-5 \\
\hline GO:0030154 BP & Cell differentiation & 43 & $6.9 \mathrm{E}-5$ \\
\hline GO:0045944 BP & Positive regulation of transcription from RNA polymerase II promoter & 50 & $1.6 \mathrm{E}-4$ \\
\hline GO:0005634 CC & Nucleus & 208 & 2.4E-4 \\
\hline GO:0009887 BP & Organ morphogenesis & 12 & $2.9 \mathrm{E}-4$ \\
\hline GO:0035925 MF & mRNA 3'-UTR AU-rich region binding & 5 & $3.0 \mathrm{E}-4$ \\
\hline GO:0008285 BP & Negative regulation of cell proliferation & 25 & $3.1 \mathrm{E}-4$ \\
\hline GO:0048709 BP & Oligodendrocyte differentiation & 7 & 3.6E-4 \\
\hline GO:0000381 BP & Regulation of alternative mRNA splicing, via spliceosome & 7 & 7.8E-4 \\
\hline GO:0000122 BP & Negative regulation of transcription from RNA polymerase II promoter & 37 & $1.1 \mathrm{E}-3$ \\
\hline GO:0031012 CC & Extracellular matrix & 19 & $1.7 \mathrm{E}-3$ \\
\hline \multicolumn{4}{|l|}{ T2DM } \\
\hline GO:0030054 CC & Cell junction & 147 & $1.4 \mathrm{E}-26$ \\
\hline GO:0030424 CC & Axon & 98 & $1.9 \mathrm{E}-26$ \\
\hline GO:0043195 CC & Terminal bouton & 46 & 8.7E-21 \\
\hline GO:0006811 BP & Ion transport & 112 & $5.1 \mathrm{E}-18$ \\
\hline GO:0005216 MF & Ion channel activity & 48 & $1.8 \mathrm{E}-14$ \\
\hline GO:0042734 CC & Presynaptic membrane & 31 & $2.4 \mathrm{E}-14$ \\
\hline GO:0034765 BP & Regulation of ion transmembrane transport & 42 & $3.8 \mathrm{E}-14$ \\
\hline GO:0008021 CC & Synaptic vesicle & 39 & $3.6 \mathrm{E}-13$ \\
\hline GO:0005244 MF & Voltage-gated ion channel activity & 40 & $5.0 \mathrm{E}-13$ \\
\hline GO:0005509 MF & Calcium ion binding & 111 & $2.9 \mathrm{E}-12$ \\
\hline GO:0045211 CC & Postsynaptic membrane & 54 & $3.6 \mathrm{E}-13$ \\
\hline GO:0005886 CC & Plasma membrane & 509 & $3.5 \mathrm{E}-12$ \\
\hline GO:0006810 BP & Transport & 227 & $1.1 \mathrm{E}-11$ \\
\hline GO:0007268 BP & Chemical synaptic transmission & 44 & $1.4 \mathrm{E}-11$ \\
\hline GO:0008076 CC & Voltage-gated potassium channel complex & 27 & $2.2 \mathrm{E}-10$ \\
\hline GO:0007269 BP & Neurotransmitter secretion & 17 & $3.6 \mathrm{E}-11$ \\
\hline GO:0000775 CC & Chromosome, centromeric region & 38 & $7.1 \mathrm{E}-11$ \\
\hline GO:0006813 BP & Potassium ion transport & 34 & $1.1 \mathrm{E}-9$ \\
\hline GO:0005267 MF & Potassium channel activity & 26 & $1.4 \mathrm{E}-9$ \\
\hline GO:0005887 CC & Integral component of plasma membrane & 150 & $5.7 \mathrm{E}-10$ \\
\hline
\end{tabular}

GO, gene ontology; BP, biological process; MF, molecular function; CC, cellular component; T1DM, type 1 diabetes mellitus; T2DM, type 2 diabetes mellitus.

Table III. Coincident enriched Kyoto Encyclopedia of Genes and Genomes pathways in T1- and T2DM.

\begin{tabular}{lccrr}
\hline & \multicolumn{2}{c}{ T1DM } & & T2DM \\
\cline { 2 - 5 } Term & Gene count & P-value & Gene count \\
\hline mmu04010:MAPK signaling pathway & 19 & $7.5 \mathrm{E}-4$ & 29 & $3.2 \mathrm{E}-2$ \\
mmu04512:ECM-receptor interaction & 10 & $1.4 \mathrm{E}-3$ & 15 & $6.9 \mathrm{E}-3$ \\
mmu04390:Hippo signaling pathway & 11 & $1.8 \mathrm{E}-2$ & 24 & $1.2 \mathrm{E}-3$ \\
mmu04360:Axon guidance & 9 & $4.5 \mathrm{E}-2$ & $3.8 \mathrm{E}-2$ \\
\hline
\end{tabular}

T1DM, type 1 diabetes mellitus; T2DM, type 2 diabetes mellitus. 
Table IV.Enriched Kyoto Encyclopedia of Genes and Genomes pathways in T1- and T2DM, respectively.

\begin{tabular}{|c|c|c|}
\hline Term & $\begin{array}{l}\text { Gene } \\
\text { count }\end{array}$ & P-value \\
\hline \multicolumn{3}{|l|}{ T1DM } \\
\hline mmu04010:MAPK signaling pathway & 19 & $7.5 \mathrm{E}-4$ \\
\hline mmu04512:ECM-receptor interaction & 10 & $1.4 \mathrm{E}-3$ \\
\hline mmu05202:Transcriptionalmisregulation in cancer & 14 & $1.7 \mathrm{E}-3$ \\
\hline mmu05200:Pathways in cancer & 24 & $2.5 \mathrm{E}-3$ \\
\hline mmu05205:Proteoglycans in cancer & 15 & $3.8 \mathrm{E}-3$ \\
\hline mmu04310:Wnt signaling pathway & 11 & $1.1 \mathrm{E}-2$ \\
\hline mmu05214:Glioma & 7 & $1.4 \mathrm{E}-2$ \\
\hline mmu05222:Small cell lung cancer & 8 & $1.4 \mathrm{E}-2$ \\
\hline mmu04015:Rap1 signaling pathway & 14 & $1.4 \mathrm{E}-2$ \\
\hline mmu04330:Notch signaling pathway & 6 & $1.7 \mathrm{E}-2$ \\
\hline mmu04914:Progesterone-mediated & 8 & $1.7 \mathrm{E}-2$ \\
\hline \multicolumn{3}{|l|}{ oocyte maturation } \\
\hline mmu04390:Hippo signaling pathway & 11 & $1.8 \mathrm{E}-2$ \\
\hline mmu00100:Steroid biosynthesis & 4 & $1.9 \mathrm{E}-2$ \\
\hline mmu05218:Melanoma & 7 & $2.1 \mathrm{E}-2$ \\
\hline mmu04614:Renin-angiotensin system & 5 & $2.2 \mathrm{E}-2$ \\
\hline mmu04520:Adherens junction & 7 & $2.3 \mathrm{E}-2$ \\
\hline mmu04510:Focal adhesion & 13 & $2.5 \mathrm{E}-2$ \\
\hline mmu04913:Ovarian steroidogenesis & 6 & $3.0 \mathrm{E}-2$ \\
\hline mmu04360:Axon guidance & 9 & 4.5E-2 \\
\hline mmu05210:Colorectal cancer & 6 & $4.6 \mathrm{E}-2$ \\
\hline mmu05212:Pancreatic cancer & 6 & 4.9E-2 \\
\hline \multicolumn{3}{|l|}{ T2DM } \\
\hline mmu05033:Nicotine addiction & 19 & $1.6 \mathrm{E}-10$ \\
\hline mmu04080:Neuroactive ligand-receptor interaction & 49 & $1.4 \mathrm{E}-7$ \\
\hline mmu04723:Retrograde endocannabinoid signaling & 23 & $8.5 \mathrm{E}-6$ \\
\hline mmu04721:Synaptic vesicle cycle & 17 & $1.2 \mathrm{E}-5$ \\
\hline mmu05032:Morphine addiction & 20 & $6.7 \mathrm{E}-5$ \\
\hline mmu04514:Cell adhesion molecules (CAMs) & 27 & $2.4 \mathrm{E}-4$ \\
\hline mmu04724:Glutamatergic synapse & 21 & $4.3 \mathrm{E}-4$ \\
\hline mmu04024:cAMP signaling pathway & 30 & $4.9 \mathrm{E}-4$ \\
\hline mmu04923:Regulation of lipolysis in adipocytes & 13 & $1.1 \mathrm{E}-3$ \\
\hline mmu04390:Hippo signaling pathway & 24 & $1.2 \mathrm{E}-3$ \\
\hline mmu04020:Calcium signaling pathway & 26 & $2.7 \mathrm{E}-3$ \\
\hline mmu04972:Pancreatic secretion & 17 & $3.8 \mathrm{E}-3$ \\
\hline mmu00561:Glycerolipid metabolism & 12 & $4.2 \mathrm{E}-3$ \\
\hline mmu04911:Insulin secretion & 15 & $5.6 \mathrm{E}-3$ \\
\hline mmu04727:GABAergic synapse & 15 & $6.2 \mathrm{E}-3$ \\
\hline mmu04512:ECM-receptor interaction & 15 & $6.9 \mathrm{E}-3$ \\
\hline mmu04261:Adrenergic signaling in cardiomyocytes & 20 & $2.1 \mathrm{E}-2$ \\
\hline mmu04022:cGMP-PKG signaling pathway & 22 & $2.2 \mathrm{E}-2$ \\
\hline mmu04810:Regulation of actin cytoskeleton & 26 & $2.3 \mathrm{E}-2$ \\
\hline mmu04726:Serotonergic synapse & 18 & $2.4 \mathrm{E}-2$ \\
\hline mmu04725:Cholinergic synapse & 16 & $2.6 \mathrm{E}-2$ \\
\hline mmu04728:Dopaminergic synapse & 18 & $2.8 \mathrm{E}-2$ \\
\hline mmu04610:Complement and coagulation cascades & 12 & $3.0 \mathrm{E}-2$ \\
\hline mmu04010:MAPK signaling pathway & 29 & $3.2 \mathrm{E}-2$ \\
\hline mmu04970:Salivary secretion & 12 & 3.3E-2 \\
\hline mmu04360:Axon guidance & 17 & $3.8 \mathrm{E}-2$ \\
\hline mmu04062:Chemokine signaling pathway & 23 & $4.6 \mathrm{E}-2$ \\
\hline
\end{tabular}

T1DM, type 1 diabetes mellitus; T2DM, type 2 diabetes mellitus.
Table V. Top 10 proteins with relatively high connectivity degrees in the protein-protein interaction networks for T1- and T2DM.

\begin{tabular}{clllllll}
\hline \multicolumn{3}{c}{ T1DM } & & & \multicolumn{3}{c}{ T2DM } \\
\cline { 1 - 3 } \cline { 5 - 6 } Rank & Protein & Degree & & Rank & Protein & Degree \\
\cline { 1 - 3 } 1 & Lrrk1 & 76 & & 1 & Top2a & 171 \\
2 & Trp53 & 65 & & 2 & Cdk1 & 144 \\
3 & Actb & 60 & & 3 & Plk1 & 126 \\
4 & Actr1b & 42 & & 4 & Aurkb & 120 \\
5 & Fn1 & 40 & & 5 & Plk4 & 116 \\
6 & Yes1 & 38 & & 6 & Ccnb1 & 115 \\
7 & Mapk12 & 38 & & 7 & Aurka & 114 \\
8 & Prkacb & 33 & & 8 & Mki67 & 113 \\
9 & Pten & 32 & & 9 & Kif11 & 112 \\
10 & Ywhaz & 28 & & 10 & Cdc20 & 111 \\
\hline
\end{tabular}

T1DM, type 1 diabetes mellitus; T2DM, type 2 diabetes mellitus.

Regarding DPN, it has been suggested that perturbation of insulin receptor signaling due to insulin resistance may cause neurons to become more vulnerable to metabolic insults and contribute to the pathogenesis of neuropathy (31). However, how gene expression is altered under the insulin-resistant state in nerve tissue remains unclear for T2DM. In the present study, a number of unique GO terms and signaling pathways were determined for the sciatic nerve profile of T2DM mice, which may result from an insulin-resistant state. For instance, a majority of the GO terms among the top 20 enriched terms unique to T2DM were related to the biological processes associated with cell junctions, ion activity and membrane activity. Previous studies indicated that the insulin-resistant state induced phosphorylation and downregulated of the expression of certain connexin $(\mathrm{Cx})$ proteins including $\mathrm{Cx} 43$, which may constitute a potential mechanism underlying the pathogenesis of insulin resistance and its complications $(32,33)$. Furthermore, it was demonstrated that $\mathrm{Ca}^{2+}$ overload in the mitochondria caused production of superoxide and functional impairment of multiple tissues, which may result in $\beta$-cell failure and insulin resistance in target tissues, further aggravating the complications of diabetes (34). Therefore, it may be speculated that the impairment of nerve tissues under the insulin-resistant state is attributable to these DEGs in the aforementioned biological processes in T2DM.

In addition, it was apparent that the efficacy of different treatment strategies for the two types of DM may be attributed to certain DEGs. For example, treatment of obesity appeared as critical as glycemic control for preventing the development of DPN in T2DM (5). In the present study, C-C chemokine receptor 7 (Ccr7), which was among the proteins with the highest degrees of connectivity in the PPI network for T2DM, could interact with the chemokine ligand 19 (Ccl19). The Cc119-Ccr7 pathway may serve an important role in development of high-fat-induced obesity and subsequent insulin resistance (35). Therefore, it may be suggested that a potential treatment strategy is through Ccr7 targeting to alleviate insulin resistance and neuropathy in T2DM. 
Table VI. Information on the modules of the protein-protein interaction networks in T1- and T2DM.

\begin{tabular}{|c|c|c|c|c|c|}
\hline Module & Node number & P-value & $\begin{array}{l}\text { Protein with highest } \\
\text { connectivity degree (degree) }\end{array}$ & $\begin{array}{l}\text { GO term } \\
\text { number }\end{array}$ & $\begin{array}{l}\text { KEGG pathway } \\
\text { number }\end{array}$ \\
\hline \multicolumn{6}{|l|}{ T1DM } \\
\hline 1 & 15 & $9.4 \mathrm{E}-5$ & Srsf1 (22) & 18 & 0 \\
\hline \multicolumn{6}{|l|}{ T2DM } \\
\hline 1 & 125 & $4.2 \mathrm{E}-14$ & Top2a (171) & 151 & 8 \\
\hline 2 & 28 & $2.9 \mathrm{E}-10$ & Frrs11 (28) & 2 & 0 \\
\hline 3 & 24 & $8.3 \mathrm{E}-8$ & Ccr7 (46) & 79 & 5 \\
\hline 4 & 41 & $1.0 \mathrm{E}-4$ & Mapk4 (72) & 40 & 4 \\
\hline 5 & 11 & $2.8 \mathrm{E}-4$ & Gabrg2 (25) & 37 & 5 \\
\hline 6 & 32 & $3.4 \mathrm{E}-4$ & $\operatorname{Scn} 8 \mathrm{a}(52)$ & 74 & 4 \\
\hline 7 & 20 & $6.0 \mathrm{E}-4$ & Aldh1a2 (30) & 34 & 9 \\
\hline
\end{tabular}

T1DM, type 1 diabetes mellitus; T2DM, type 2 diabetes mellitus.

Limitation of the current study included the data being obtained from online databases, meaning the genetic backgrounds of the mice could vary substantially. Further genetic analyses are therefore warranted to identify genes and determine the molecular differences in neuropathy between the two types of DM based on different strains of mice.

In conclusion, the present study revealed the gene expression profiles and signaling pathways associated with the sciatic nerve in T1- and T2DM mouse models. The DEGs and signaling pathways may indicate unique biological processes and treatment strategies for the two types of DM. Further molecular biological experiments are required to validate the function of the DEGs and signaling pathways in DPN.

\section{Acknowledgements}

Not applicable.

\section{Funding}

The present study was supported by the National Natural Science Foundation of China (grant no. 81701104), the Natural Science Foundation of Guangdong Province, China (grant no. 2016A030310157), the Technology Planning Project of Guangdong Province, China (grant no. 2016A020220010) and the Technology Planning Project of Guangzhou, China (grant no. 201604020120).

\section{Availability of data and materials}

All data used and/or analyzed during this study are included in this published article.

\section{Authors' contributions}

XL designed the study and aided in drafting of the manuscript. YG and ZLQ performed the bioinformatics analysis and drafted the manuscript. DZL and GLS analyzed the data. ZQH and YCG interpreted the results and prepared the figures. XL and $\mathrm{ZQH}$ edited and revised manuscript. All authors approved the final version of the manuscript to be published.

\section{Ethics approval and consent to participate}

Not applicable.

\section{Consent for publication}

Not applicable.

\section{Competing interests}

The authors declare that they have no competing interests.

\section{References}

1. Vincent AM, Callaghan BC, Smith AL and Feldman EL: Diabetic neuropathy: Cellular mechanisms as therapeutic targets. Nat Rev Neurol 7: 573-583, 2011.

2. Tesfaye S and Selvarajah D: Advances in the epidemiology, pathogenesis and management of diabetic peripheral neuropathy. Diabetes Metab Res Rev 28 (Suppl 1): 8-14, 2012.

3. Premkumar LS and Pabbidi RM: Diabetic peripheral neuropathy: Role of reactive oxygen and nitrogen species. Cell Biochem Biophys 67: 373-383, 2013.

4. Levitt NS, Stansberry KB, Wynchank S and Vinik AI: The natural progression of autonomic neuropathy and autonomic function tests in a cohort of people with IDDM. Diabetes Care 19: 751-754, 1996.

5. Rathmann W, Ziegler D, Jahnke M, Haastert B and Gries FA: Mortality in diabetic patients with cardiovascular autonomic neuropathy. Diabet Med 10: 820-824, 1993.

6. O'Brien PD, Sakowski SA and Feldman EL: Mouse models of diabetic neuropathy. ILAR J 54: 259-272, 2014.

7. Callaghan BC, Hur J and Feldman EL: Diabetic neuropathy: One disease or two? Curr Opin Neurol 25: 536-541, 2012.

8. Sima AA and Kamiya H: Diabetic neuropathy differs in type 1 and type 2 diabetes. Ann NY Acad Sci 1084: 235-249, 2006.

9. Kamiya H, Murakawa Y, Zhang W and Sima AA: Unmyelinated fiber sensory neuropathy differs in type 1 and type 2 diabetes. Diabetes Metab Res Rev 21: 448-458, 2005.

10. Stevens MJ, Zhang W, Li F and Sima AA: C-peptide corrects endoneurial blood flow but not oxidative stress in type $1 \mathrm{BB} /$ Wor rats. Am J Physiol Endocrinol Metab 287: E497-E505, 2004.

11. Qu W, Han C, Li M, Zhang J and Li L: Revealing the underlying mechanism of diabetic nephropathy viewed by microarray analysis. Exp Clin Endocrinol Diabetes 123: 353-359, 2015.

12. He K, Lv W, Zhang Q, Wang Y, Tao L and Liu D: Gene set enrichment analysis of pathways and transcription factors associated with diabetic retinopathy using a microarray dataset. Int J Mol Med 36: 103-112, 2015. 
13. Lacroix-Fralish ML, Tawfik VL, Tanga FY, Spratt KF and DeLeo JA: Differential spinal cord gene expression in rodent models of radicular and neuropathic pain. Anesthesiology 104: 1283-1292, 2006.

14. Zhang L, Qu S, Liang A, Jiang H and Wang H: Gene expression microarray analysis of the sciatic nerve of mice with diabetic neuropathy. Int J Mol Med 35: 333-339, 2015.

15. Wiggin TD, Kretzler M, Pennathur S, Sullivan KA, Brosius FC and Feldman EL: Rosiglitazone treatment reduces diabetic neuropathy in streptozotocin-treated DBA/2J mice. Endocrinology 149: 4928-4937, 2008.

16. Pande M, Hur J, Hong Y, Backus C, Hayes JM, Oh SS, Kretzler M and Feldman EL: Transcriptional profiling of diabetic neuropathy in the BKS db/db mouse: A model of type 2 diabetes. Diabetes 60 : 1981-1989, 2011

17. Simon R, Lam A, Li MC, Ngan M, Menenzes S and Zhao Y: Analysis of gene expression data using BRB-ArrayTools. Cancer Inform 3: 11-17, 2007.

18. Huang W, Sherman BT and Lempicki RA: Systematic and integrative analysis of large gene lists using DAVID bioinformatics resources. Nat Protoc 4: 44-57, 2009.

19. Huang W, Sherman BT and Lempicki RA: Bioinformatics enrichment tools: Paths toward the comprehensive functional analysis of large gene lists. Nucleic Acids Res 37: 1-13, 2009.

20. Wang J, Ma SH, Tao R, Xia LJ, Liu L and Jiang YH: Gene expression profile changes in rat dorsal horn after sciatic nerve injury. Neurol Res 39: 176-182, 2017.

21. Yang YL, Xiang RL, Yang C, Liu XJ, Shen WJ, Zuo J, Chang YS and Fang FD: Gene expression profile of human skeletal muscle and adipose tissue of Chinese Han patients with type 2 diabetes mellitus. Biomed Environ Sci 22: 359-368.

22. Sun J, Wang D and Jin T: Insulin alters the expression of components of the Wnt signaling pathway including TCF-4 in the intestinal cells. Biochim Biophys Acta 1800: 344-351, 2010.

23. Matsumoto K and Yokoyama S: Gene expression analysis on the liver of cholestyramine-treated type 2 diabetic model mice. Biomed Pharmacother 64: 373-378.

24. Abdul-Rahman O, Sasvari-Szekely M, Ver A, Rosta K, Szasz BK, Kereszturi E and Keszler G: Altered gene expression profiles in the hippocampus and prefrontal cortex of type 2 diabetic rats. BMC Genomics 13: 81, 2012.

25. Mounier $C$ and Posner BI: Transcriptional regulation by insulin: From the receptor to the gene. Can J Physiol Pharmacol 84 713-724, 2006

26. Schuelert N, Gorodetskaya N, Just S, Doods H and Corradini L: Electrophysiological characterization of spinal neurons in different models of diabetes type 1- and type 2-induced neuropathy in rats. Neuroscience 291: 146-154, 2015.
27. Echeverry S, Shi XQ and Zhang J: Characterization of cell proliferation in rat spinal cord following peripheral nerve injury and the relationship with neuropathic pain. Pain 135: 37-47, 2008

28. Sun C, Zhang J, Chen L, Liu T, Xu G, Li C, Yuan W, Xu H and Su Z: IL-17 contributed to the neuropathic pain following peripheral nerve injury by promoting astrocyte proliferation and secretion of proinflammatory cytokines. Mol Med Rep 15: 89-96, 2017.

29. Guzen FP, de Almeida Leme RJ, de Andrade MS, de Luca BA and Chadi G: Glial cell line-derived neurotrophic factor added to a sciatic nerve fragment grafted in a spinal cord gap ameliorates motor impairments in rats and increases local axonal growth. Restor Neurol Neurosci 27: 1-16, 2009.

30. Chang-Chen KJ, Mullur R and Bernal-Mizrachi E: Beta-cell failure as a complication of diabetes. Rev Endocr Metab Disord 9: 329-343, 2008.

31. Kim B,McLean LL,Philip SS and Feldman EL:Hyperinsulinemia induces insulin resistance in dorsal root ganglion neurons. Endocrinology 152: 3638-3647, 2011.

32. Bian O, Zhang H, Guan Q, Sun Y and Zeng D: High-dose insulin inhibits gap junction intercellular communication in vascular smooth muscle cells. Mol Med Rep 12: 331-336, 2015.

33. Takenaka T, Inoue T, Okada H, Ohno Y, Miyazaki T, Chaston DJ, Hill CE and Suzuki H: Altered gap junctional communication and renal haemodynamics in Zucker fatty rat model of type 2 diabetes. Diabetologia 54: 2192-2201, 2011.

34. Ly LD, Xu S, Choi SK, Ha CM, Thoudam T, Cha SK, Wiederkehr A, Wollheim CB, Lee IK and Park KS: Oxidative stress and calcium dysregulation by palmitate in type 2 diabetes. Exp Mol Med 49: e291, 2017.

35. Sano T, Iwashita M, Nagayasu S, Yamashita A, Shinjo T, Hashikata A, Asano T, Kushiyama A, Ishimaru N, Takahama Y, et al: Protection from diet-induced obesity and insulin resistance in mice lacking CCL19-CCR7 signaling. Obesity (Silver Spring) 23: $1460-1471,2015$

This work is licensed under a Creative Commons Attribution-NonCommercial-NoDerivatives 4.0 International (CC BY-NC-ND 4.0) License. 